\title{
Recent metal-catalysed asymmetric hydroaminations of alkenes.
}

\author{
Christophe Michon*a,b ${ }^{\text {, }}$ Marc-Antoine Abadie ${ }^{\mathrm{a}, \mathrm{b}}$, Florian Medina ${ }^{\mathrm{a}, \mathrm{b}}$, \\ Francine Agbossou-Niedercorn*a,b
}

Dedicated to Prof. John A. Gladysz at the occasion of the $65^{\text {th }}$ birthday.

${ }^{a}$ Univ. Lille, CNRS, Centrale Lille, ENSCL, Univ. Artois, UMR 8181 - UCCS - Unité de Catalyse et Chimie du Solide, F-59000 Lille, France.

${ }^{b}$ ENSCL, UCCS-CCM-MOCAH, (Chimie-C7) CS 90108, 59652 Villeneuve d'Ascq Cedex, France. christophe.michon@ensc-lille.fr, Tel.: (+)33320436863; francine.agbossou@ensc-lille.fr,Tel.: (+)33320434927

\begin{abstract}
The selective and asymmetric addition of amines across alkene $\mathrm{C}=\mathrm{C}$ bonds (hydroamination of alkenes) is one of the most important tool to synthesise chiral amines. Though this reaction remains a challenge due to its high energy gap and the difficult control of the enantioselection, some chiral organometallic catalysts can provide promising activities and enantioselectivities. This minireview highlights the recent use of chiral organometallic complexes in homogeneous catalysed asymmetric hydroaminations of non-activated alkenes from 2013 to 2016.
\end{abstract}

Keywords: hydroamination, alkenes, metals, asymmetric catalysis.

\section{Contents:}

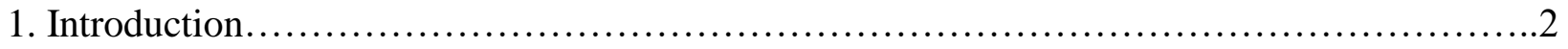

2. Group 2 / alkaline-earth catalysts - magnesium ........................................

3. Group 3 / rare-earth catalysts - lanthanides............................................ 3

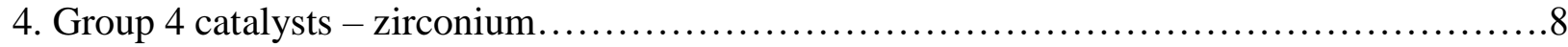

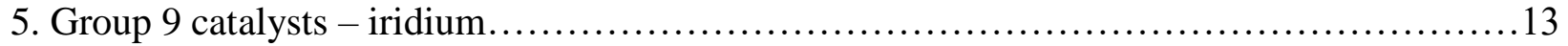

6. Group 10 catalysts - palladium................................................ 14

7. Group 11 catalysts - copper and gold........................................... 15

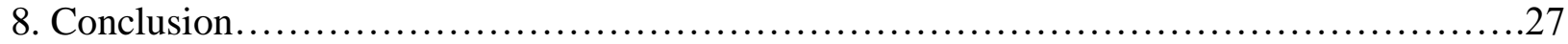

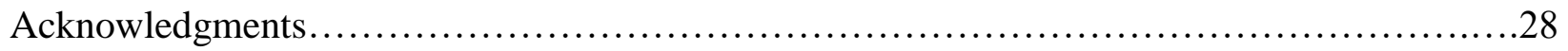

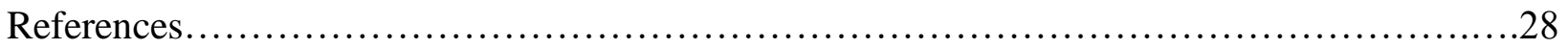




\section{Introduction}

The formation of $\mathrm{C}-\mathrm{N}$ bonds through highly efficient and selective reactions has been a long standing goal for organic chemists. Indeed, due to their broad and various applications, amines are prominently present in bulk and fine chemicals, as racemic or optically pure compounds [1]. Hence, the preparation of enantioenriched amines through stereoselective routes has been a first priority along the past decades and various approaches like reductive aminations, alcohol aminations and transaminations have been studied [1]. Hydroamination reaction which corresponds to the addition of a nitrogen and hydrogen atoms across an unsaturated carboncarbon bond constitutes the most direct and atom economical synthetic route to amines. It can provide either Markovnikov (branched) product, either anti-Markovnikov (linear) product, either both. The reaction regioselectivity can be influenced by alkene and amine substituents, by the formation of a privileged ring (intramolecular reactions) or by the use of a catalyst implying a preferred reaction pathway [2]. Along the past decades, great achievements have been reported for inter- and intramolecular processes applying catalysts based on transition metals, lanthanides and actinides as well as on main group elements and acid catalysts [2]. However, if hydroamination reactions were successful through the proper combination of catalysts, alkenes and amines of various nucleophilies, a general approach providing a highly selective synthetic methodology applicable to a wide range of internal and terminal non-activated alkenes remains still challenging. Nonetheless, important achievements have been reported along the past years and need to be addressed in this review. Indeed, several original chiral organometallic catalysts have allowed reaching promising catalytic activities and enantioselectivities for the synthesis of various chiral amines. Moreover, a seminal and broadly applicable synthetic method has recently introduced the amine fragment by following an electrophilic pathway (formal hydroamination) rather than the conventional nucleophilic one. Through an amine umpolung, i.e. a reversal of the amine polarity, this new single step pathway could circumvent the energy barrier resulting from the electronic repulsion between the amine lone pair and the $\pi$-bond of the olefin. If asymmetric hydroamination had been reviewed in the past [2], we would like to highlight herein the latest achievements in the field of asymmetric hydroamination of non-activated alkenes covering researches reported from 2013 to 2016 and not reviewed yet. Metal catalysed asymmetric bisamination of alkenes using di-tbutyldiaziridinone and analogues are not covered herein [3]. 


\section{Group 2 / alkaline-earth catalysts - magnesium}

Though alkaline-earths may not be considered strictly as metals, the application of organo-magnesium or calcium species led in the past to several significant achievements on asymmetric and racemic hydroaminations of alkenes [4]. Recently, Dorta, Harder et al. reported the synthesis of a magnesium amidophosphine complex based on (S,S)-trans-9,10-dihydro-9,10ethanoanthracene-11,12-diamine (ANDEN) and its application for the intramolecular hydroamination of 2,2'-diphenyl-pent-4-ene-amine (Scheme 1) [5]. When a primary amine substrate was involved, the hydroamination reaction proceeded smoothly to afford in good yield the corresponding pyrrolidine at room temperature provided $5 \mathrm{~mol} \%$ of catalyst was used. As the result of a possible Schlenk-type ligand-redistribution process [4d], the resulting enantioselectivities were low and a change to a secondary amine substrate yielded the corresponding pyrrolidine in trace amounts only.
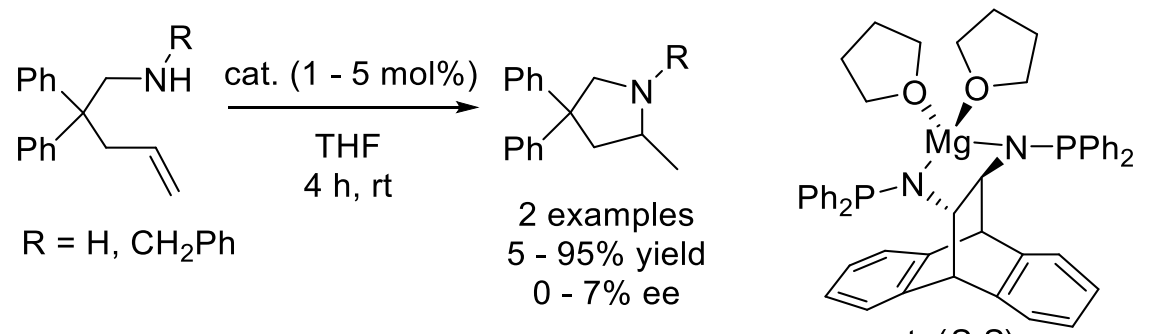

cat. $(S, S)$

Scheme 1. Magnesium catalysed intramolecular hydroamination of alkenes by Dorta et al.

\section{Group 3 / rare-earth catalysts - lanthanides.}

In 2013, Hultzsch et al. reported $C_{1}$-symmetric yttrium and lutetium aminodiolate complexes which were active and selective catalysts for the intramolecular hydroamination of alkenes in rather mild conditions (Scheme 2) [6]. By comparison to previous catalysts based on binaphtholates or other polydentate ligands [7], the required catalyst loadings were higher due to the presence of an additional amine donor site in the ligand. Primary amino-pentenes and hexenes reacted in good yields and low to high enantioselectivities. The bulkiness of the $\mathrm{R}^{6}$ and $\mathrm{R}^{8}$ catalyst substituents was critical to reach high enantioselectivities and lutetium species were more selective than yttrium ones though requiring longer reaction times. In addition, similar lanthanum complexes did not exhibit improved catalytic activities. It was worth to note the prepared lanthanide complexes were also active catalysts for the intermolecular hydroamination 
of unactivated alkenes at high temperatures and long reaction times using a large excess of alkene as respect to the primary amine reagent. Yields of the secondary amine products were average and enantioselectivities were low to average.

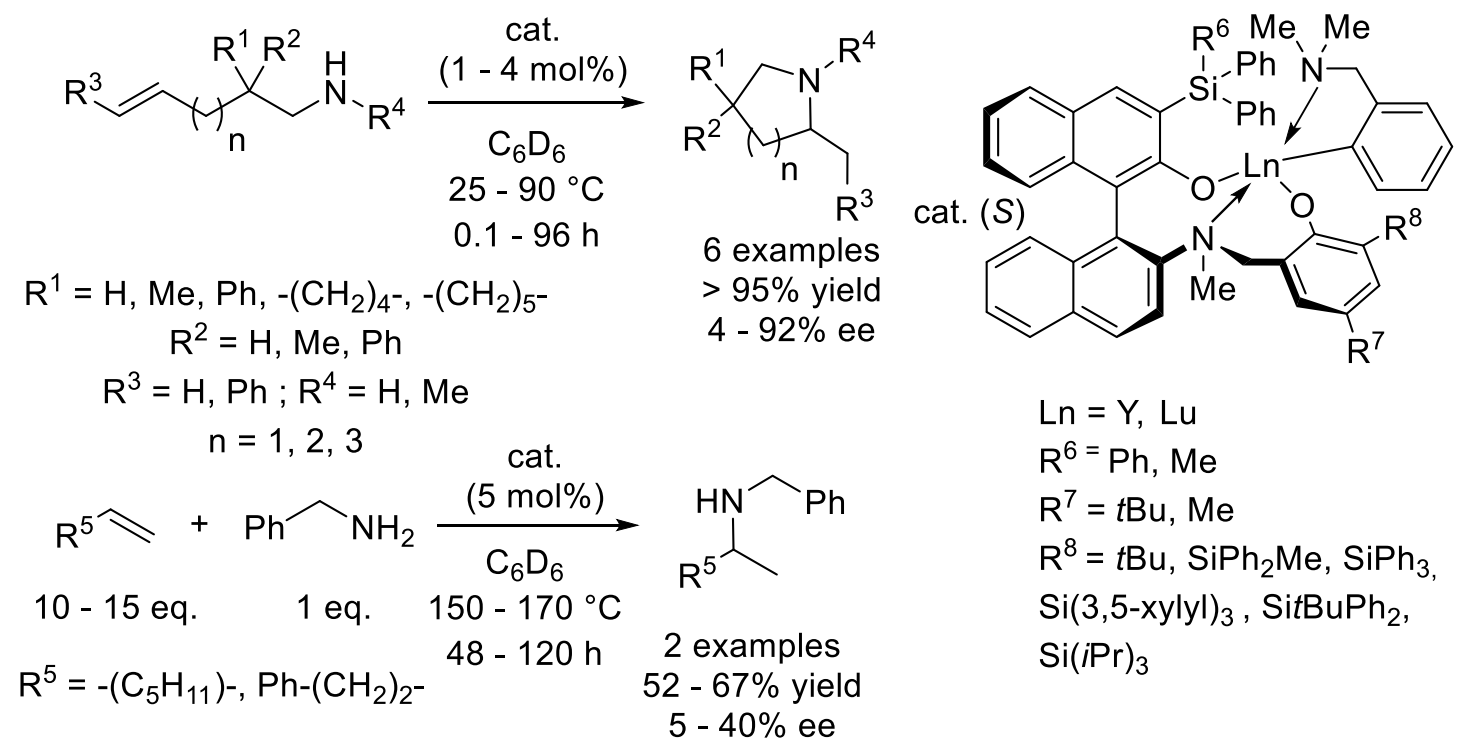

Scheme 2. Lanthanide catalysed intermolecular hydroamination of alkenes by Hultzsch et al.

The synthesis and characterisation of chiral alkyl or amido yttrium complexes from $\mathrm{N}$-silyl- or $N$-cyclopentyl-substituted binaphthylamido ligands was reported by Hannedouche, Trifonov et al. (Scheme 3) [8]. These neutral species proved to be active catalysts for the intramolecular hydroamination of amino-alkenes yielding to pyrrolidine and piperidine derivatives in high yields with low to good enantioselectivities. Independently of the catalyst, the presence of $\mathrm{LiCl}$ proved to lead to higher asymmetric inductions through a heterobimetallic cooperative effect [9].

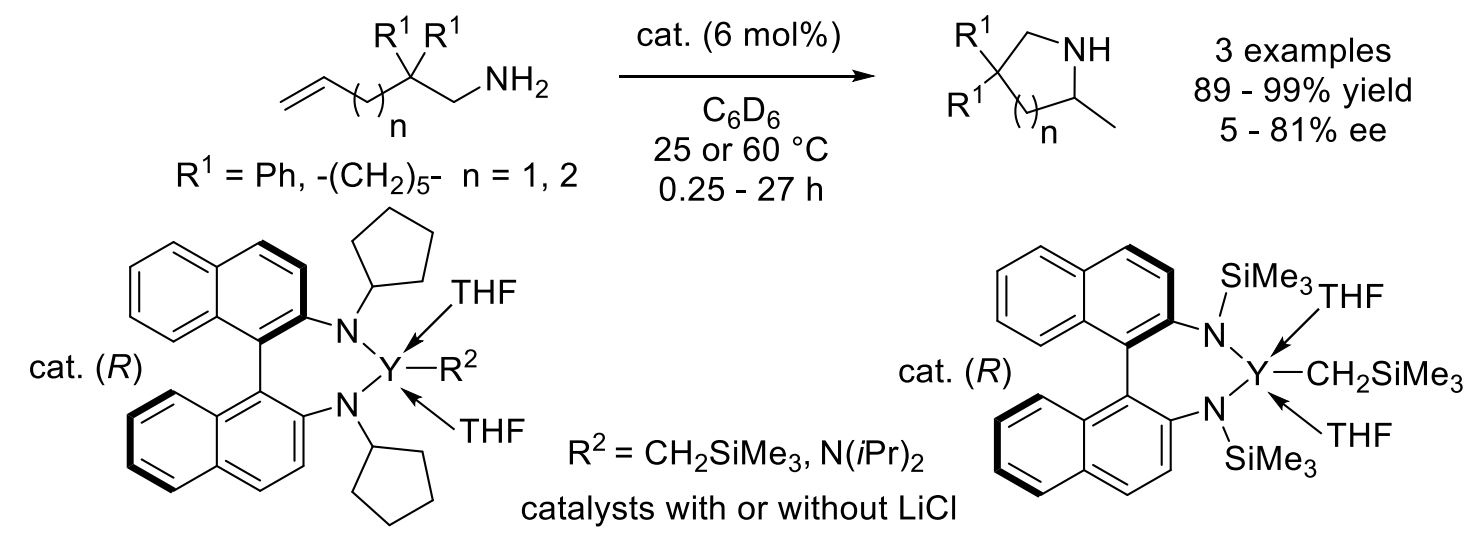

Scheme 3. Lanthanide catalysed intermolecular hydroamination of alkenes by Schulz et al. 
However, if these already known interactions between a catalyst and $\mathrm{LiCl}$ could be evidenced in solution by NMR experiments, they could not be confirmed at the solid state. A close structureactivity relationship was noticed for the catalysts and allowed some improvements, ligands bearing alkyl substituents being more active than those with silyl groups.

To further extend the scope of their binaphthylamido alkyl yttrium catalysts, Hannedouche, Schulz et al. studied the tandem intermolecular anti-Markovnikov - intramolecular Markovnikov hydroamination of $C$-(1-allylcyclohexyl)methylamine and 2-vinylpyridine (Scheme 4) [10]. If inter- and intramolecular products were obtained in various amounts, the tandem product could be obtained with a high yield and average enantioselectivity by using the cyclopentyl substituted catalyst. Depending on the reaction temperature and the nature of the catalyst, a retrohydroamination reaction could occur implying the conversion of the tandem product into the intramolecular product (step 2).

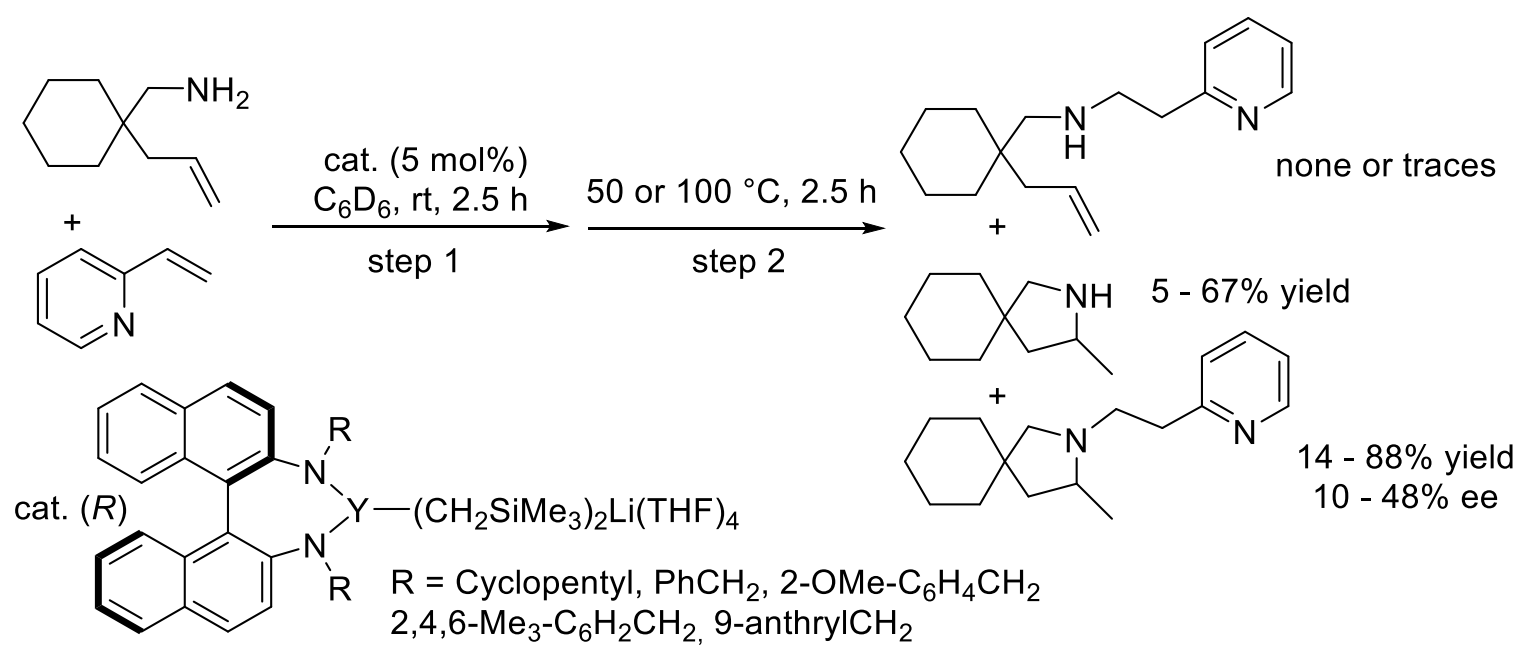

Scheme 4. Yttrium catalysed intermolecular and intramolecular hydroamination of alkenes

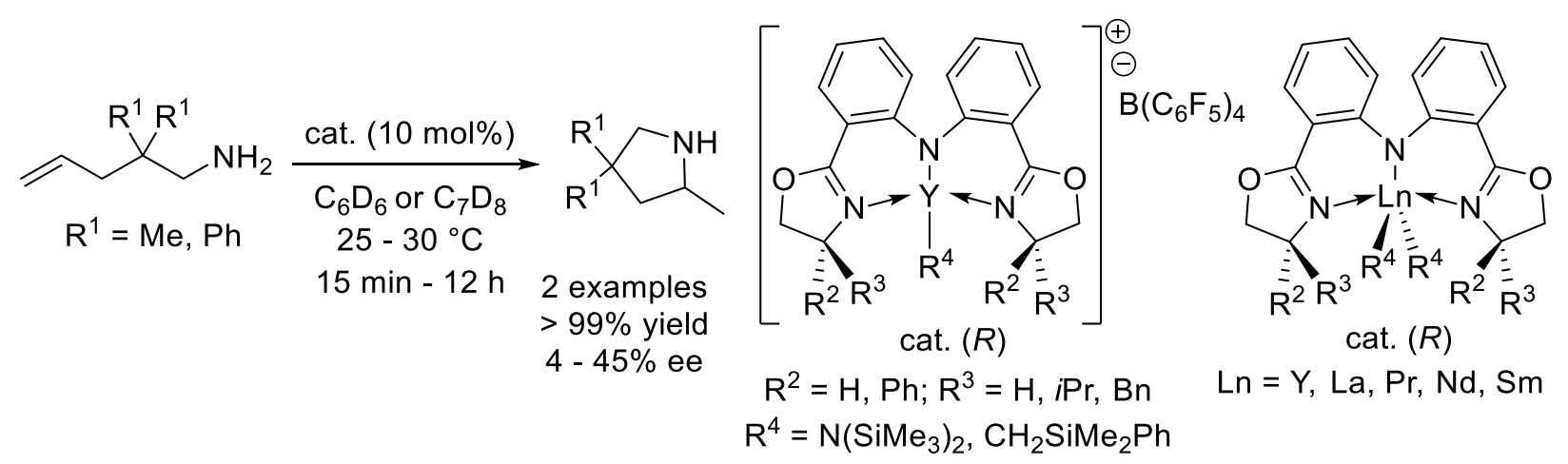

Scheme 5. Lanthanide catalysed intermolecular hydroamination of alkenes by Ward et al. 
In 2014, Ward, Mountford et al. reported the synthesis and deep characterisation of several chiral lanthanide complexes based on $(R)$-BOPA ligand and studied some intramolecular aminoalkene hydroaminations (Scheme 5) [11]. On the whole, conversions were high and enantioselectivities were low to average. If bulky yttrium amide complexes were unreactive within various reaction conditions, less hindered alkyl derivatives afforded pyrrolidine products in high conversions. Due to a strong Thorpe-Ingold effect [12], substrates bearing methyl groups were far less reactive then aminoalkenes bearing phenyl groups. However, the use of larger lanthanides allowed better conversions though enantioselectivities remained low. If catalysts substituted by phenyl groups were often the most selective, a solvent change from benzene to toluene resulted also in a significant increase of the enantioselections.

In two consecutive publications, Livinghouse et al. reported the syntheses and applications of several neutral and anionic lanthanum(III) and yttrium(III) chelating diamide complexes for intramolecular aminoalkene hydroaminations (Scheme 6) [13].

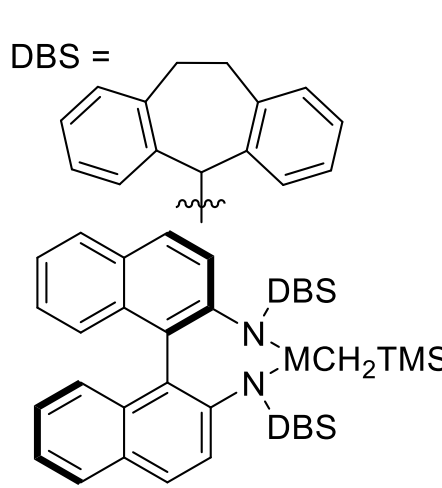

cat. $(R) \mathrm{M}=\mathrm{Y}, \mathrm{La}, \mathrm{Sc}, \mathrm{Lu}$ $47-88 \%$ ee<smiles>[R5]N(S)c1ccc2ccccc2c1-c1c(N([R5])CCCCCCC)ccc2ccccc12</smiles>

cat. $(R) \mathrm{M}=\mathrm{Y}, \mathrm{La}, \mathrm{Sc}, \mathrm{Lu}$ $47-78 \%$ ee
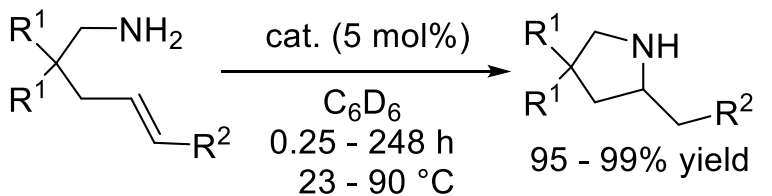

$\mathrm{R}^{1}=\mathrm{Me}$, allyl

$\mathrm{R}^{2}=\mathrm{H}$, aryl, heteroaryl

$95-99 \%$ yield

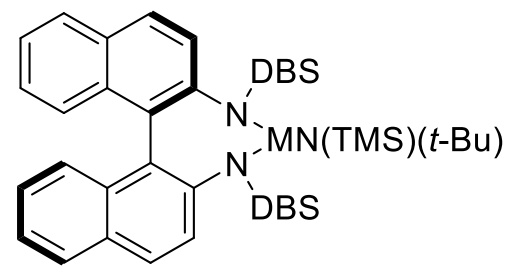

cat. $(R) \mathrm{M}=\mathrm{Y}$, La

$48-87 \%$ ee

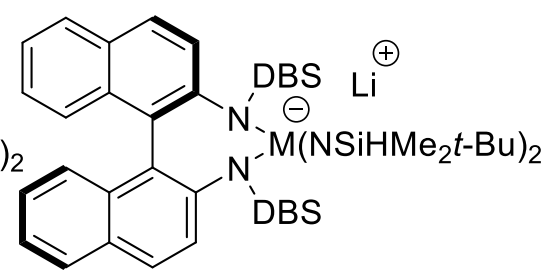

cat. $(R) \mathrm{M}=\mathrm{Y}$, La $33-81 \%$ ee

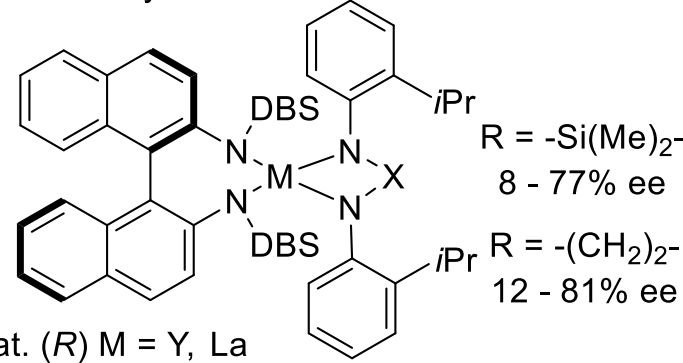

cat. $(R) \mathrm{M}=\mathrm{Y}, \mathrm{La}$

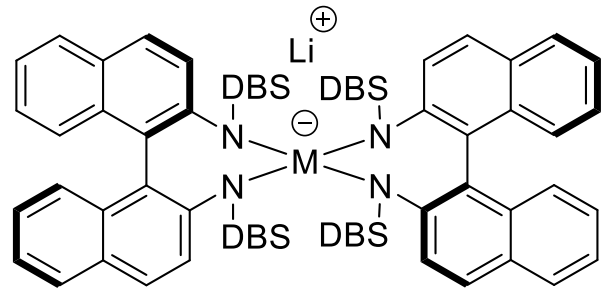

cat. $(R) \mathrm{M}=\mathrm{Y}$, La

$52-82 \%$ ee

Scheme 6. Lanthanide catalysed intermolecular hydroamination of alkenes by Livinghouse et al.

Yields in pyrrolidines were high and enantioselectivities were low to high. Authors noticed lanthanum(III) catalysts were more efficient than the yttrium(III) ones providing better activities 
as well as some of the highest enantioselectivities for intramolecular alkene hydroamination. Surprisingly, any $\mathrm{LiCl}$ remaining from the neutral complex syntheses was found to suppress both the rates and the enantioselectivities obtained with the described neutral yttrium(III) complexes when compared to the lanthanum(III) counterparts. To circumvent such drawback, some neutral amide complexes could be prepared using the active bases $\mathrm{La}[\mathrm{N}(\mathrm{TMS})(t \mathrm{Bu})]_{3}$ and $\mathrm{Y}[\mathrm{N}(\mathrm{TMS})(t \mathrm{Bu})]_{3}$. Anionic catalysts were also prepared but, by comparison to the neutral species, they gave little advantages in term of activities or selectivities and required the presence of Li cations to reach high enantioselectivities.

The synthesis of an yttrium complex based on a novel chiral silicon-linked tridentate amidoindenyl ligand was reported by Yang et al. (Scheme 7) [14].
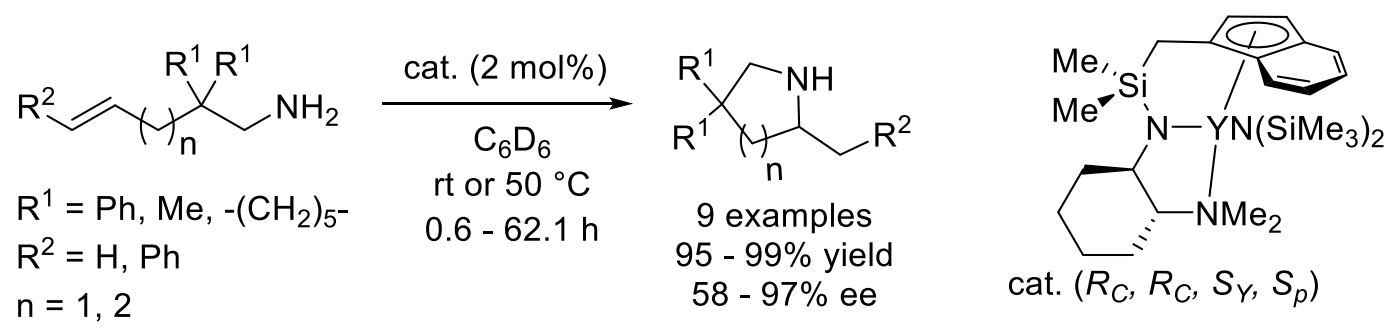

Scheme 7. Lanthanide catalysed intermolecular hydroamination of alkenes by Yang et al.

The resulting species catalysed the intramolecular hydroamination of aminoalkenes with high yields and average to high enantioselectivities. If the authors noticed a decrease of enantioselection at sub-ambient temperatures, the reactions performed well at room temperature for the synthesis of pyrrolidines and piperidines or for challenging substrates like 1,2disubstituted olefins. If this new yttrium complex has three chiral elements: the chiral 1,2cyclohexanediamine $(R, R)$, the chiral yttrium centre $(S)$ and the chiral indene ring $\left(S_{\mathrm{p}}\right)$, the chiral planarity of the indene ring may epimerize as suggested by the X-ray structure of a corresponding erbium complex. If the influence of the ligand chirality on the actual catalytic process remains unclear to the authors, a putative indenyl effect, i.e. a ring slippage between $\eta^{5}$ and $\eta^{3}$ coordination modes on the cyclopentadienyl unit [15], may lead, at least, to 2 catalytic species and result in a non-linear effect which would be negative according the authors results. It is worth to note indenyl effects were previously observed on other zirconium and titanium complexes [16]. 
In 2016, Hou et al. reported the first enantioselective intermolecular hydroamination of cyclopropenes with various amines by using chiral half-sandwich catalysts based on samarium, yttrium and lanthanum (Scheme 8) [17].

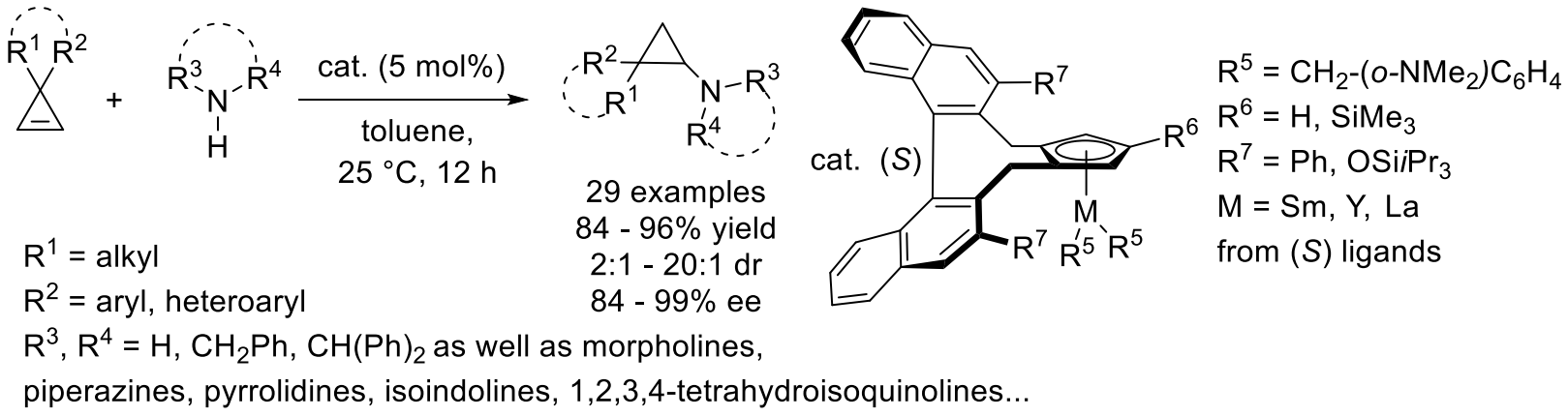

Scheme 8. Lanthanide catalysed intermolecular hydroamination of cyclopropenes by Hou et al.

A variety of chiral $\alpha$-aminocyclopropane derivatives was prepared in high yields and excellent stereoselectivities (diastereo- and enantioselectivities) at room temperature. The authors noticed a significant influence of the metal and the cyclopentadienyl based ligand on the catalyst activity and stereoselectivity. If samarium complexes were highly effective provided the ligands were functionalised by phenyl groups, yttrium or lanthanum precursors had to be preferred in few specific cases. Indeed, the reactions involving acyclic amines resulted in higher enantioselectivities with a lanthanum based catalyst built on a bulkier substituted cyclopentadienyl ligand. Moreover, when a primary amine like benzhydrylamine was involved, an yttrium catalyst had to be preferred to reach a high enantioselectivity. According to the authors, further modifications of the ligands and the ligand/metal combination shall modify the steric and electronic environment and lead to the development of more selective, efficient, and broadly applicable catalysts.

In 2016, Roesky et al. reported the syntheses and applications of several enantiomerically pure amidinate neutral complexes of lanthanum(III) and yttrium(III) for intramolecular hydroaminations of alkenes (Scheme 9) [18a]. All reactions proceeded with high to quantitative yields at mild temperatures. For 5 membered ring products, enantioselectivities were low to average, alkyl catalysts based on scandium and lutetium being the most selective. However, longer reaction times were required for amino-alkenes bearing methyl groups due to a significant Thorpe-Ingold effect [12]. The same trend was observed for 6-membered ring products along 
with lower enantioselectivities.
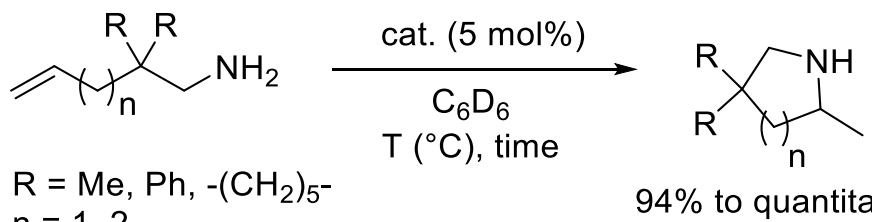$$
\mathrm{n}=1,2
$$

$94 \%$ to quantitative yield

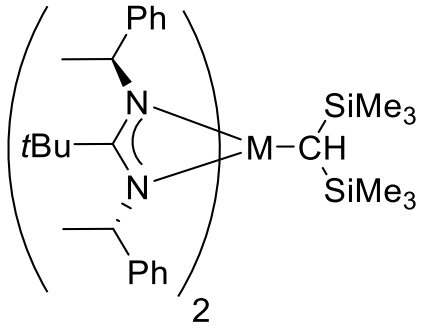

cat. $(S, S) \mathrm{M}=\mathrm{Sc}, \mathrm{Y}, \mathrm{Lu}$

5 examples

$20-40{ }^{\circ} \mathrm{C}, 1-78 \mathrm{~h}$

$5-48 \%$ ee

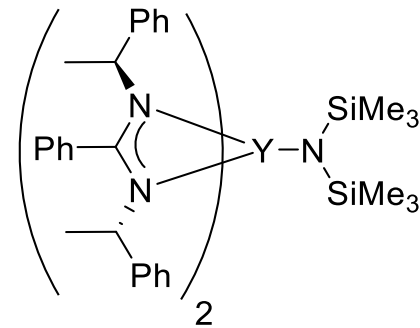

cat. $(S, S)$

1 example, $\mathrm{R}=\mathrm{Ph}$

$20^{\circ} \mathrm{C}, 9 \mathrm{~h}$ $10 \%$ ee

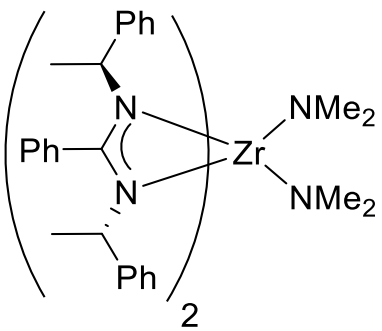

cat. $(S, S)$

3 examples

$140{ }^{\circ} \mathrm{C}, 6-31 \mathrm{~h}$

$0-9 \%$ ee

Scheme 9. Intramolecular hydroamination of alkenes catalysed by enantiopure amidinate metal complexes by Roesky et al.

\section{Group 4 catalysts - Zirconium.}

In 2013, Roesky et al. reported the synthesis of an enantiomerically pure benzamidinate zirconium complex and its further catalytic application for intramolecular hydroamination of alkenes (Scheme 9) [18b]. Though good yields were observed at high temperatures for 5 and 6membered ring products, enantioselectivities remained low.

Latter, Blechert, Roesky et al. used bimetallic copper- or zinc-zirconium complexes based on new salen-type ligands to catalyse the asymmetric intramolecular hydroamination of alkenes (Scheme 10) [19]. If the zinc or copper mononuclear complexes were not active catalysts, the combination of the bromine-free salen-type ligand with $\mathrm{Zn}(\mathrm{Me})_{2}$ and $\mathrm{Zr}\left(\mathrm{NMe}_{2}\right)_{4}$ provided one of the most active catalytic system affording piperidines with high conversions and enantioselectivities. Though the catalyst was generated in-situ and its structure was not determined, authors assumed $\mathrm{Zr}\left(\mathrm{NMe}_{2}\right)_{4}$ could react with the $\mathrm{NH}$ function of the phenylethyl moiety of the zinc metalloligand to release two molecules of $\mathrm{Me}_{2} \mathrm{NH}$ and form a $\mathrm{Zr}$-amido species. 

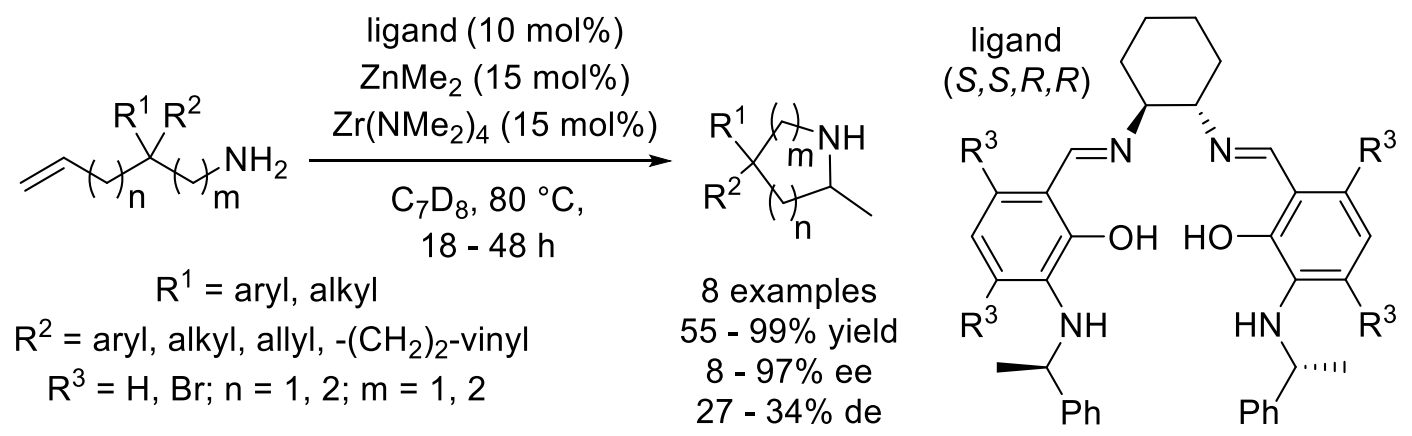

Scheme 10. Zirconium catalysed intramolecular hydroamination of alkenes by Blechert et al.

In 2013, Sadow et al. reported the synthesis and characterisation of cyclopentadienylbis(oxazolinyl)borate-supported zirconium, hafnium and titanium diamide complexes (Scheme 11) [20]. If all of these species catalysed the asymmetric intramolecular hydroamination of alkenes, zirconium based catalysts proved to be the most interesting. Various functionalised pyrrolidines, azepanes and indolines could be prepared at room temperature or below in high yields and enantioselectivities. If most of the reactions were straightforward, some reagents like aminoheptene or internal alkene derivatives proved to require much longer times to be achieved. Furthermore, catalyst activity and selectivity were found to strongly depend on the ligand structure which could be improved through modifications of the modular oxazolinylborate platform. The reaction mechanism was investigated through various kinetic, synthetic and stereochemistry experiments. The reaction required a primary amine substrate which remained unchanged in the presence of a zirconium monoamido complex or a cyclopentadienylmono(oxazolinyl)borato zirconium diamide. First, two substrates and one catalyst shall interact reversibly until an irreversible step occurred. According primary isotope effects, the activation step had a low enthalpic barrier and implied the split of a N-H bond to lead to a six-centered, concerted transition state for $\mathrm{C}-\mathrm{N}, \mathrm{C}-\mathrm{H}$ and $\mathrm{Zr}-\mathrm{N}$ bond formation and $\mathrm{N}-\mathrm{H}$ bond cleavage involving two amido-alkene ligands. Then, a zirconium imido-alkene species bound to the cyclized product could be formed. Subsequent interaction of the zirconium species with a new substrate shall allow the protonation and release of the product as well as the generation of a new zirconium diamide species ready for another reaction. It was worth to note the reaction rate diminished at high substrate concentrations suggesting that a zirconium species containing an additional substrate molecule may result in a reversible inhibition. 


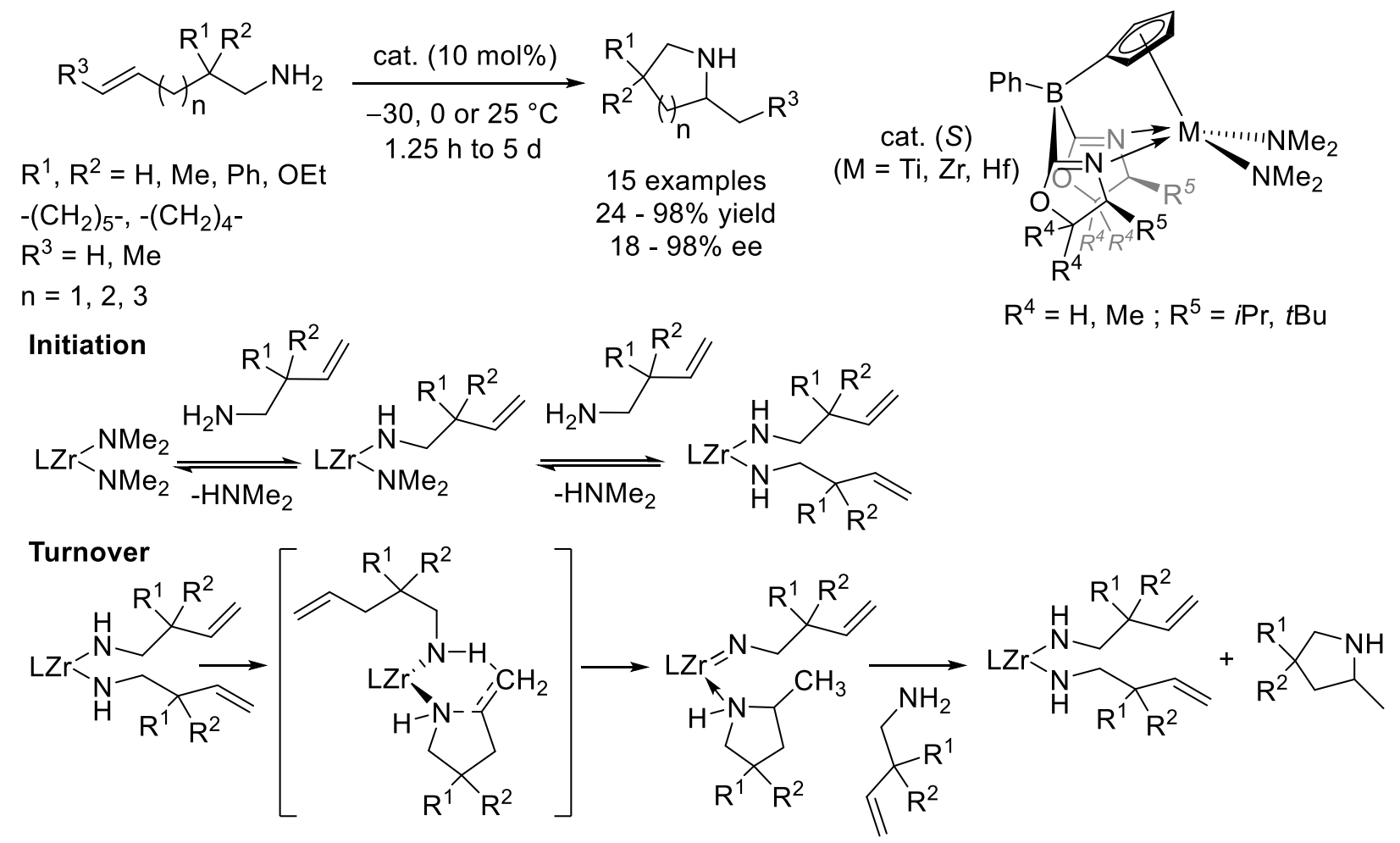

Scheme 11. Zirconium catalysed intramolecular hydroamination of alkenes by Sadow et al.

Later, Sadow et al. applied further their zirconium catalyst based on a cyclopentadienylbis(oxazolinyl)borate ligand to the desymmetrization of symmetrical aminodialkenes through enantioselective hydroamination (Scheme 12) [21]. Typically, a reaction resulted in the formation of four isomers as enantiomeric pairs of two diastereomers with one stereocentre provided by $\mathrm{C}-\mathrm{N}$ bond formation and a second one by the desymmetrization of a prochiral quaternary centre under control of the catalyst and the applied reaction conditions. Indeed, a cis-product was favoured at ambient or subambient temperature along with low initial concentrations of aminodialkene substrate whereas a trans-product was provided at low temperatures and high concentrations. Furthermore, under the latter conditions, the addition of propylamine was shown to enhance the trans-selectivity while decreasing the catalytic rate. On the whole, if such changes of reaction conditions strongly influenced the diastereoselectivities, the enantioselectivities were not significantly affected. Hence, various optically enriched five-, six-, and seven-membered cyclic amines (i.e. 1,2-dehydropyrrolidines, piperidines and azepanes) were obtained from the starting aminodialkenes in high yields, cis-trans ratios and enantioselectivities without any bicyclized products detected. Finally, the authors noticed 
deuteration of the substrate amine function enhances the stereoselectivity of the enantioselective and diastereoselective processes.

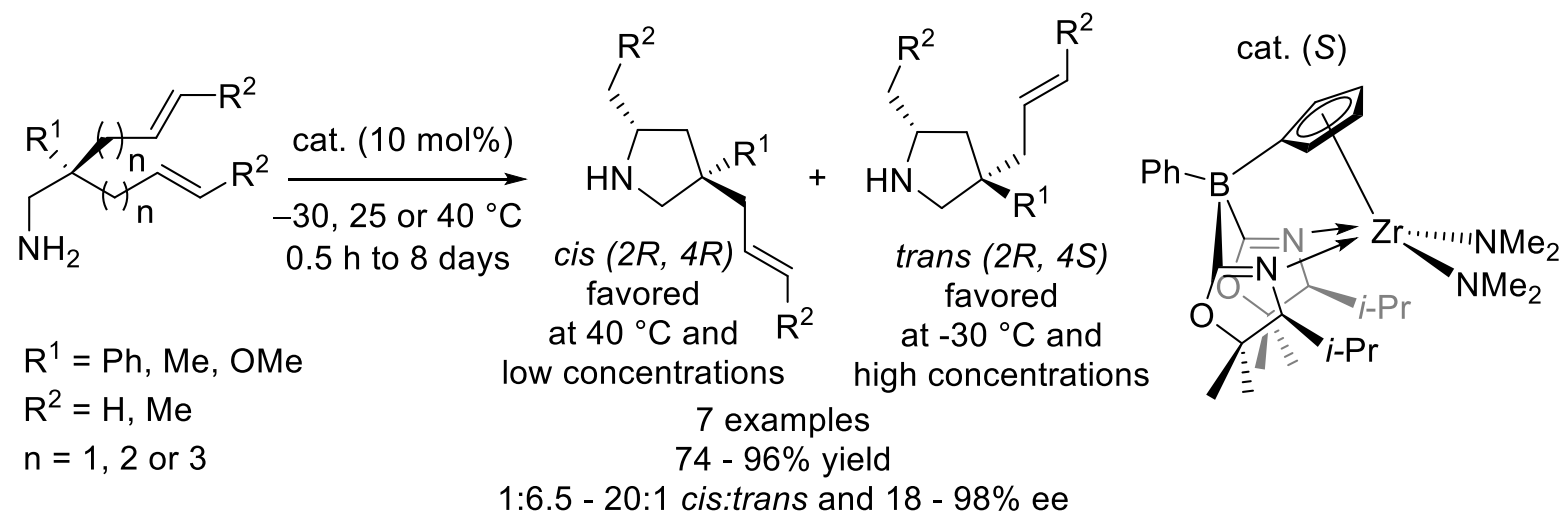

Scheme 12. Zirconium catalysed intramolecular hydroamination of symmetrical aminodialkenes

In parallel, zirconium(IV) catalysed intramolecular hydroamination of alkenes with primary amines was reported by Schafer et al. (Scheme 13) [22].

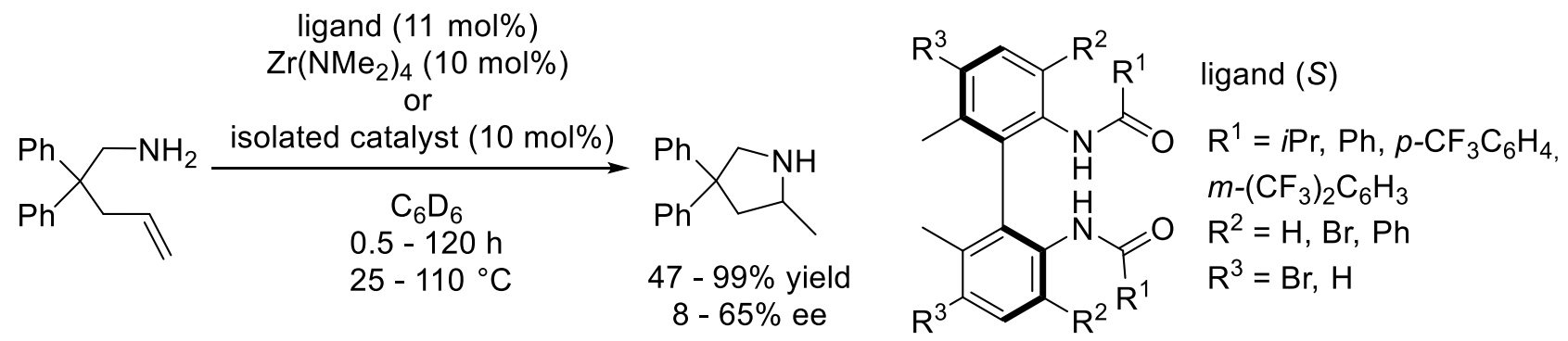

Scheme 13. Zirconium catalysed intramolecular hydroamination of alkenes by Schafer et al.

Several zirconium complexes were prepared using chiral bis(amidate) ligands based on a 3,3'substituted biphenyldiamine backbone. Overall, the use of isolated or in-situ generated catalysts led to the same results. At $110^{\circ} \mathrm{C}$, yields were usually quantitative and enantioselectivities were modest to average. A decrease of the temperature resulted in much slower reactions but slightly higher enantiomeric excesses. If the substituents at the 3,3'-positions of the biphenyl backbone were critical in promoting reactivity and enantioselectivity, X-ray structure determinations and NMR studies highlighted several possible coordination modes, $\kappa^{2}, \kappa^{3}$ and $\kappa^{4}$-dimeric species being observed. Hence, the coordination of the ligands to the zirconium appeared fluxional to the detriment of the enantioselectivity of the intramolecular hydroamination reactions. Authors 
assumed a proper ligand design may result in a configurationally stable $\kappa^{4}$-monomeric catalyst and a higher asymmetric induction.

A similar fluxional behaviour was observed by Xie and al. with a first zirconium catalyst (Scheme 14) [23].
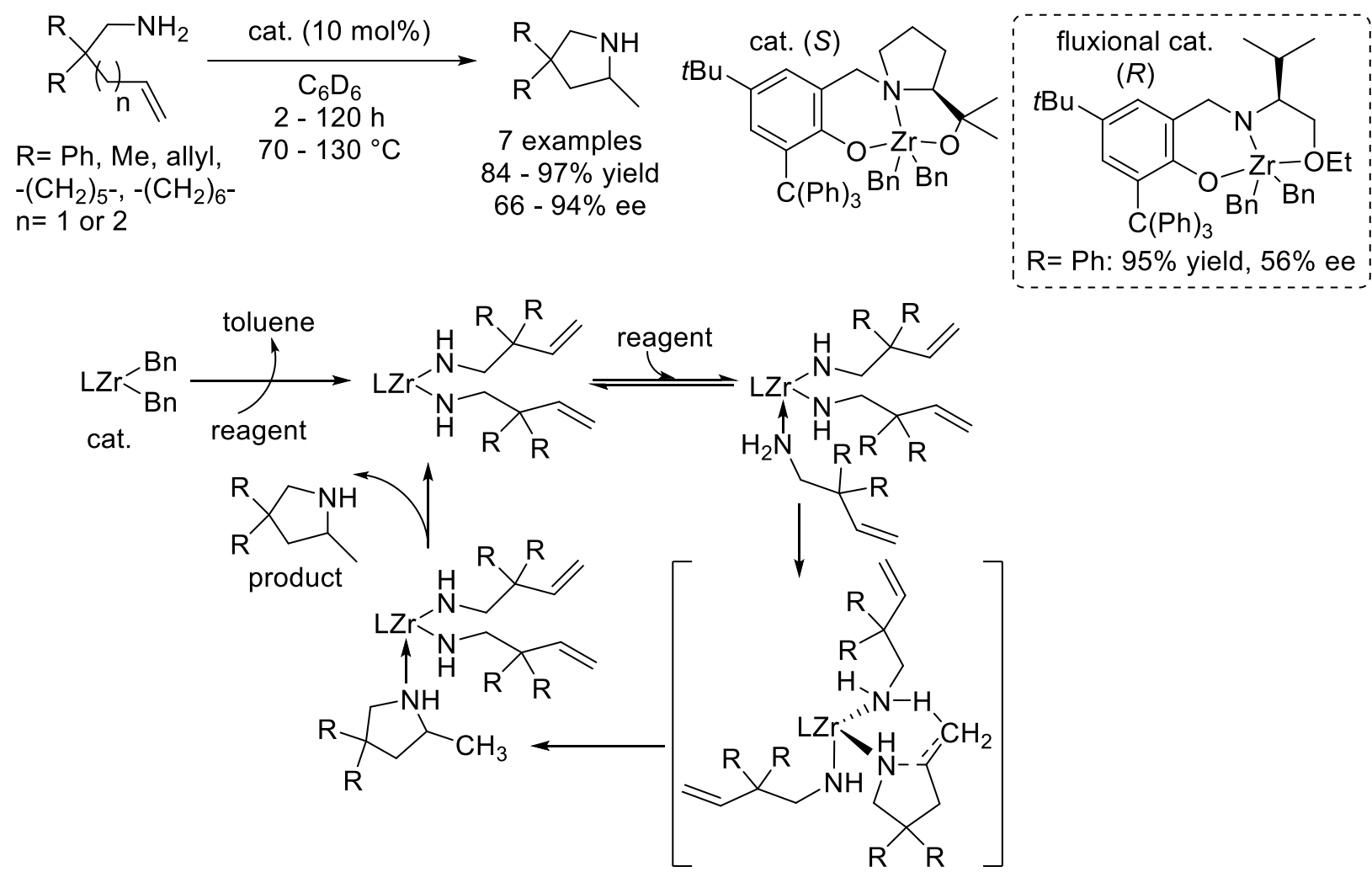

Scheme 14. Zirconium catalysed intramolecular hydroamination of alkenes by Xie et al.

The lability of the ligand $O$-ethyl group was thought to disfavour the chiral induction along intramolecular hydroamination of alkenes, the enantioselectivity being average. Authors prepared a catalyst with a new ligand backbone to allow only covalent bonds between the zirconium centre and the two oxygen atoms. The resulting catalyst was efficient for the intramolecular hydroamination reaction of various primary amino-alkenes with high yields and average to high enantioselectivities. Due to some Thorpe-Ingold effects [12], reaction time and temperature needed to be optimised for each substrate. The reaction mechanism which was studied in details through kinetics, synthesis of intermediates and control experiments proved to be similar to those already reported. If a first-order dependence on both catalyst and substrate concentrations was evidenced, a primary kinetic isotopic effect was observed when the primary 
amine was deuterated suggesting the catalytic system shall proceed via a zirconium-imido intermediate. The other parameters obtained with the kinetic study evidenced a highly organised transition state. Otherwise, a strict linear relationship between the ee values of reaction product and those of precatalyst was observed ruling out any non-linear effect and suggesting the catalyst species was monomeric. Because the deuterated pyrrolidine enantiomeric excesses were higher than the protonated products ones, it was clear an $\mathrm{N}-\mathrm{H}$ (or $\mathrm{N}-\mathrm{D}$ ) bond was involved in the stereochemistry-determining step and a concerted $\mathrm{C}-\mathrm{H} / \mathrm{C}-\mathrm{N}$ bond forming mechanism could be supported. Hence, according the authors, initial aminolysis of the precatalyst by excess substrates shall liberate toluene to give a monomeric species. A third substrate could bind reversibly to the $\mathrm{Zr}$ atom and intramolecular hydroamination took place via irreversible $\mathrm{C}-\mathrm{H}$ and $\mathrm{C}-\mathrm{N}$ bond formation through a highly ordered transition state. A further dissociation of neutral pyrrolidine regenerated the active catalyst to complete the catalytic cycle.

\section{Group 9 catalysts - Iridium.}

The first iridium(I) catalysed intramolecular asymmetric hydroamination of alkenes was reported by Dorta et al. (Scheme 15) [24].

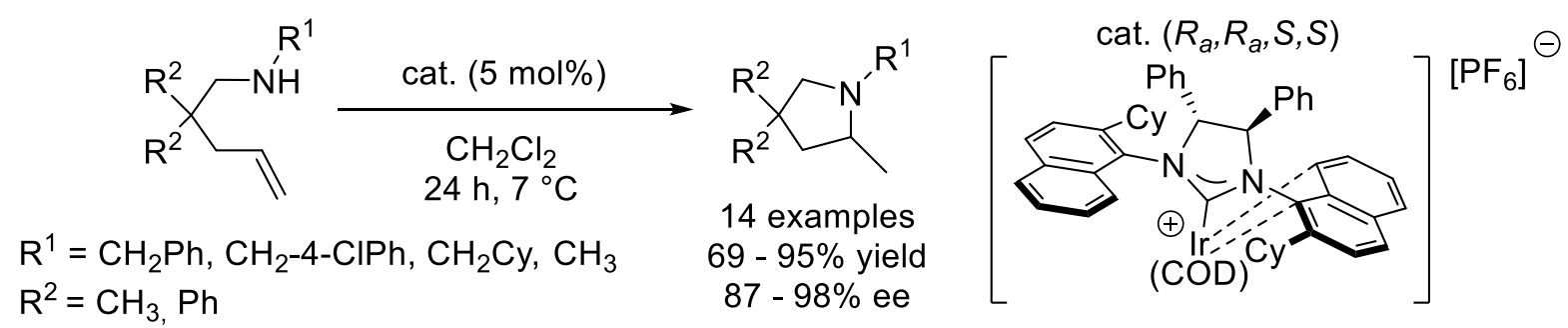

Scheme 15. Iridium catalysed intramolecular hydroamination of alkenes

The quadrant theory was applied to NHC ligands by restricting the flexibility of the $N$ substituents using functionalised napthyl side chains. This enabled a unique coordination mode of the iridium which bound to the carbene centre and to two carbons of the naphtyl substituent. As a result of an efficient transfer of the chiral information, high enantiomeric excesses were obtained for various alkyl substituted $N$-alkenyls without any significant Thorpe-Ingold effect, yields being good.

In parallel, Harder, Dorta et al. reported an iridium(I) complex able to catalyse the 
hydroamination of norbornene with anilines (Scheme 16) [5]. In the presence of a co-catalytic amount of $\mathrm{MgPh}_{2}, \mathrm{LDA}$ or $\mathrm{PhLi}$, one of the $\mathrm{N}-\mathrm{H}$ bond of anilines could be activated and the addition products exo-(2-arylamino)bornanes formed in good yields but low enantioselectivities. If the precise role of the co-catalyst was unclear, separate experiments confirmed in-situ prepared $\mathrm{Mg}$ - and Li-anilides did not catalyse the reactions even under prolonged heating.
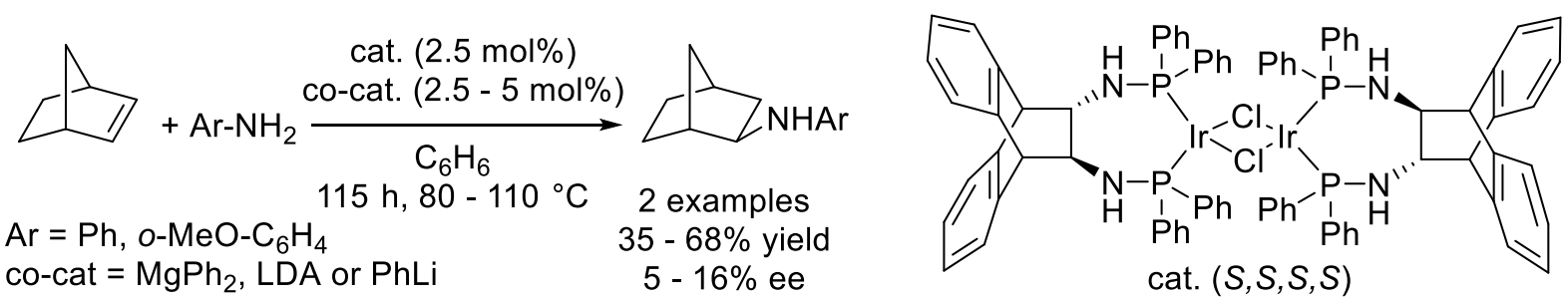

Scheme 16. Iridium catalysed intermolecular hydroamination of norbornene

\section{Group 10 catalysts - Palladium.}

In 2015, Liu et al. reported the Markovnikov intermolecular formal hydroamination of styrenes with $N$-fluorobenzenesulfonimide (NFSI) and butanol as a proton source using a pyridineoxazoline based palladium(II) catalyst (Scheme 17) [25].

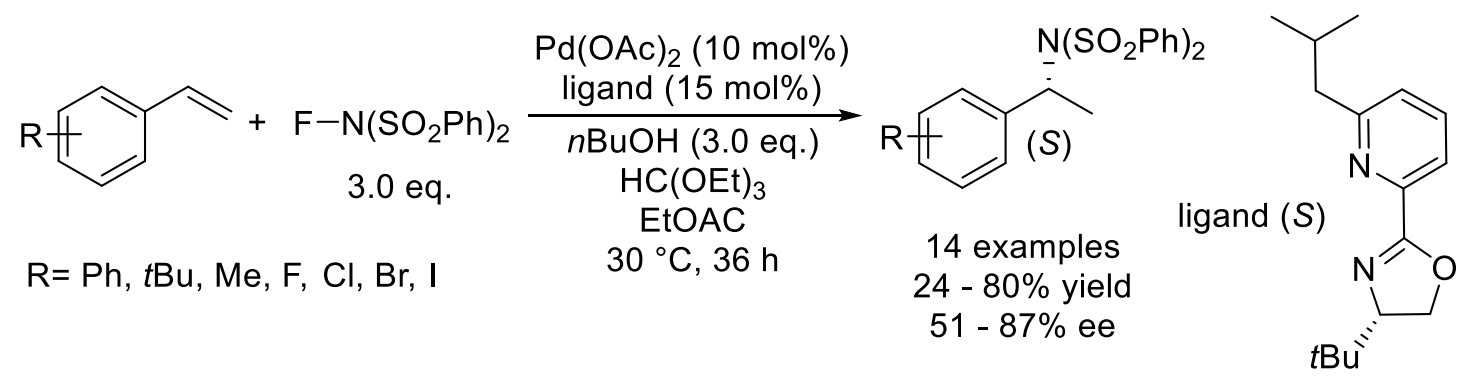

Scheme 17. Palladium catalysed intermolecular formal hydroamination of styrenes

Various chiral branched amides were obtained with excellent regioselectivities and average to high enantioselectivities. A preliminary mechanistic study using deuterium labelled styrene and alcohol revealed deuterium incorporations on either alkene carbon indicating a migratory insertion of styrene into the palladium hydride species was involved in the reaction. Though the asymmetric induction may suffer from such phenomenon, the catalyst displayed a good enantioselective control. According the authors, the catalytic system may be improved by using a 
true pseudo $C_{2}$-symmetric ligand.

The same year, Zhang et al. reported the asymmetric intramolecular hydroamination of amino1,3-dienes using a $P$-stereogenic PNP pincer-Pd complex (Scheme 18) [26].

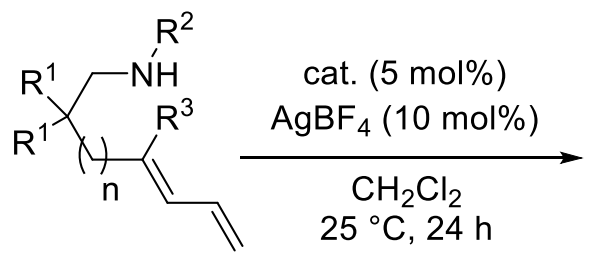

$\mathrm{R}^{1}=\mathrm{Ph}, \mathrm{Me}, \mathrm{H},-\left(\mathrm{CH}_{2}\right)_{3^{-}},-\left(\mathrm{CH}_{2}\right)_{4^{-}}$,

$-\left(\mathrm{CH}_{2}\right)_{5^{-}},-\left(\mathrm{CH}_{2}\right)_{6}-, \mathrm{C}_{4} \mathrm{H}_{4}$
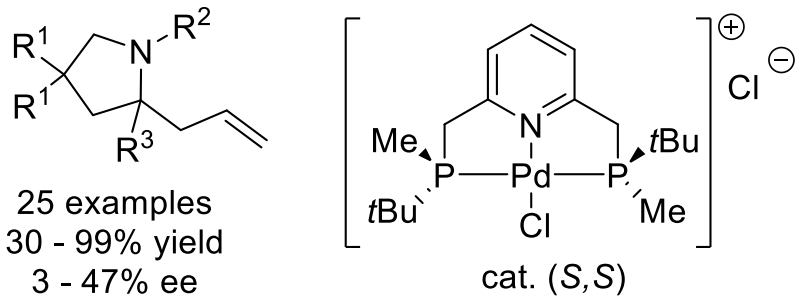

$\mathrm{R}^{2}=2,4-\mathrm{Me}_{2} \mathrm{C}_{6} \mathrm{H}_{4} \mathrm{SO}_{2}, \mathrm{CBz}$

$\mathrm{R}^{3}=\mathrm{H}, \mathrm{Me}$

Scheme 18. Palladium catalysed intramolecular hydroamination of dienes

By operating at room temperature for 24 hours, allyl-type chiral pyrrolidine derivatives were obtained regioselectively in low to good yields and with moderate to average enantioselectivities for $(S)$-enantiomers, the 2,4-dimethylbenzenesulfonyl amine protecting group affording the highest chiral induction. The authors suggested the reaction mechanism would imply a square planar $\pi$-complex intermediate. The cationic palladium(II) catalyst would bind selectively to the terminal $\mathrm{C}=\mathrm{C}$ double bond of the amino-1,3-diene, the substrate skeleton being located near the methyl substituent of the phosphorus atom to avoid the bulky tbutyl groups which occupied two of the four quadrants. The resulting intermediate would undergo the intramolecular nucleophilic addition of the amine to lead to a $\eta^{1}$-allylpalladium which would be further cleaved and protonated to release the allyl-type pyrrolidine and the catalyst. The reaction centres (e.g the free alkene and amine) being rather far from the chiral phosphorous, authors argued bulkier phosphine substituents shall help improving the enantioselectivity of that intramolecular hydroamination reaction.

\section{Group 11 catalysts - Copper and Gold.}

\subsection{Copper catalysts.}

In order to address regioselective issues associated to the intermolecular hydroamination of alkenes bearing a variety of substituents, Miura et al. developed a copper catalytic system (10 
mol\%) performing a formal hydroamination of styrenes with hydrosilanes and $O$-benzoyl hydroxylamines in the presence of a base in excess (Scheme 19) [27].
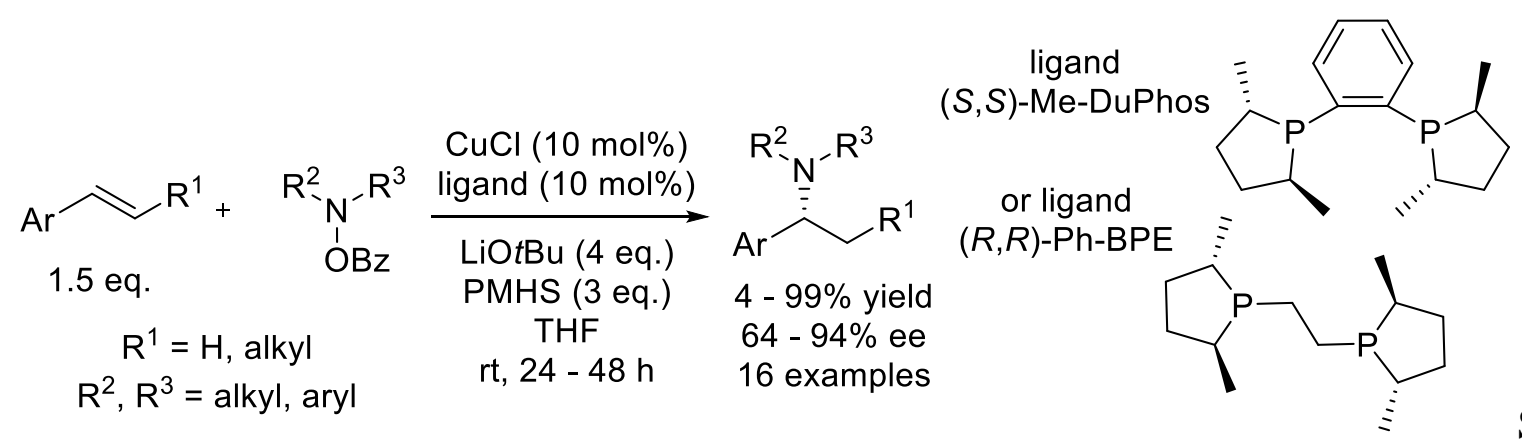

Schem

e 19. Copper-hydride catalysed formal hydroamination of alkenes by Miura et al.

This electrophilic amination (umpolung) involved an active copper-hydride species and an electrophilic amine transfer reagent. After insertion of the styrene substrate by hydrocupration, the reaction with the amination reagent afforded the hydroamination product and a copper species to reactivate for a further cycle. Mechanistic aspects are presented below (vide infra). The authors demonstrated the efficiency and high regioselectivity of the reaction for a large array of styrene substrates including $\beta$-substituted ones. The use of chiral diphosphines allowed carrying out an enantioselective amination, which provided amines with enantiomeric excesses in a $66-94 \%$ range. However, such reaction could not be considered as a strict hydroamination due to its electrophilic pathway and the resulting by-products (e.g. alkoxysilanes and benzoate salts).

At the same time, based on their past interest for the application of copper-hydride in asymmetric reactions [28], Buchwald et al. reported also a similar and efficient copper based catalytic system for both the formal anti-Markovnikov hydroamination of terminal aliphatic alkenes and the asymmetric synthesis of branched amines from styrenes (up to > 99\% ee) (Scheme 20) [29]. 


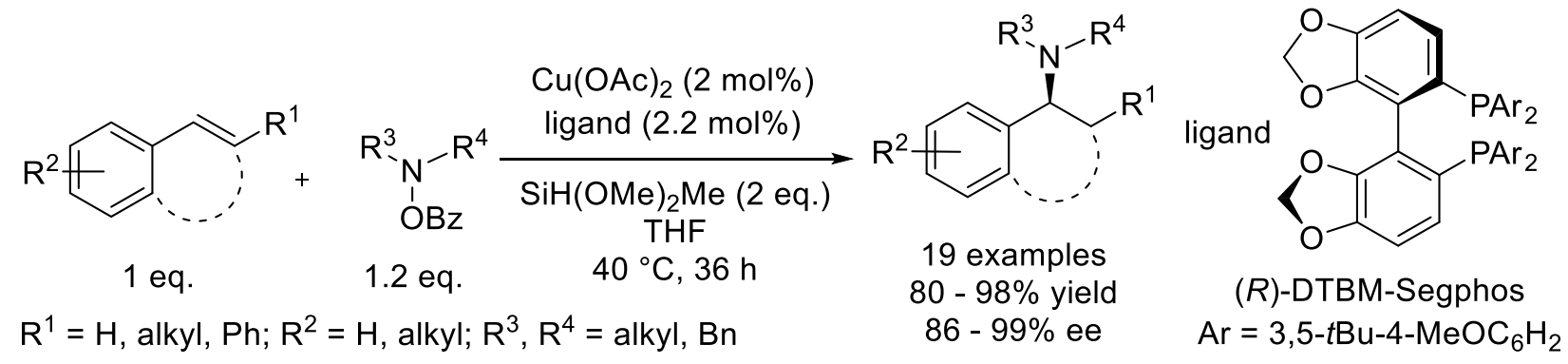

Scheme 20. Copper-hydride catalysed formal hydroamination of alkenes by Buchwald et al.

Under optimised conditions, the reactions performed well at room temperature in the presence of diethoxymethylsilane (DEMS). By comparison to Miura et al., the reactions didn't require the addition of a base and were operated with a lower catalyst loading ( 1 or 2 mol\%) obtained from the combination of $\mathrm{Cu}(\mathrm{OAc})_{2}$ and a chiral diphosphine. Though the reactions were not strict hydroaminations due to their electrophilic pathway and their alkoxysilane by-products, they were highly regioselective generating a $\mathrm{C}-\mathrm{N}$ bond at the $\alpha$-position of styrenes and tolerated various substituted styrenes including trans- and $c i s$ - $\beta$-substitutions and $\beta$ - $\beta$-disubstitutions. It was worth to note the procedure could be scaled up, $10 \mathrm{mmol}$ of $\mathrm{PhCH}=\mathrm{CHCH}_{2} \mathrm{OMe}$ being transformed into $\mathrm{PhCH}\left(\mathrm{NBn}_{2}\right) \mathrm{CH}_{2} \mathrm{CH}_{2} \mathrm{OMe}$ with a $93 \%$ yield and a $99 \%$ ee [30].

Latter, both groups further studied the potential of this unique formal alkene hydroamination reaction involving a copper-hydride, a hydrosilane and an amine transfer reagent at fundamental or applied levels, on chiral or racemic reactions as well as on alkenes or alkynes.

First, Buchwald et al. applied the procedure reported above to the regio- and enantioselective formal hydroamination of 1,1-disubstituted olefins furnishing a practical and general synthetic method towards $\beta$-chiral amines (Scheme 21) [31].

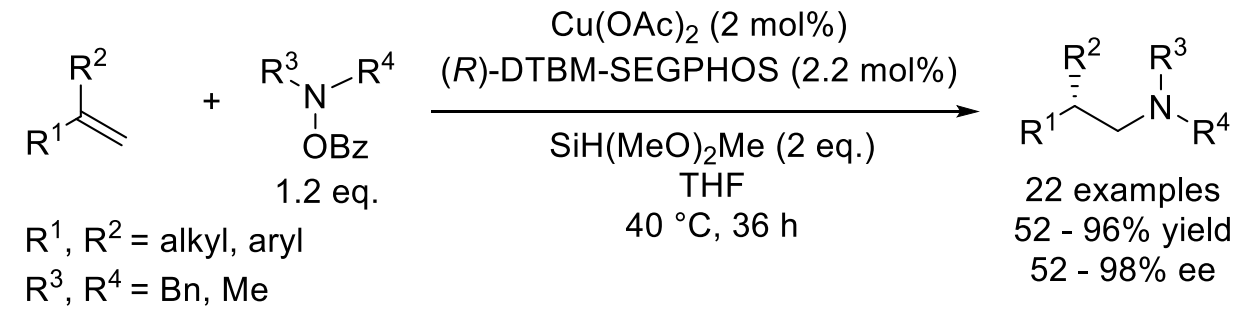

Scheme 21. Copper-hydride catalysed formal hydroamination of 1,1-disubstituted olefins.

A large array of 1,1-disubstituted alkenes was examined displaying various structural steric and 
functional group patterns. All the reported examples provided the corresponding chiral antiMarkovnikov products with good to high ee (up to $98 \%$ ee). The amine transfer reagents could incorporate heterocyclic (piperazine) and sterically hindered (2,6-tetramethyl piperidine) amines. In addition, a diastereoselective approach was also studied.

Latter, Buchwald et al. implemented also very successfully this Cu-hydride catalysed asymmetric formal hydroamination to internal olefins (Scheme 22) [32, 33].

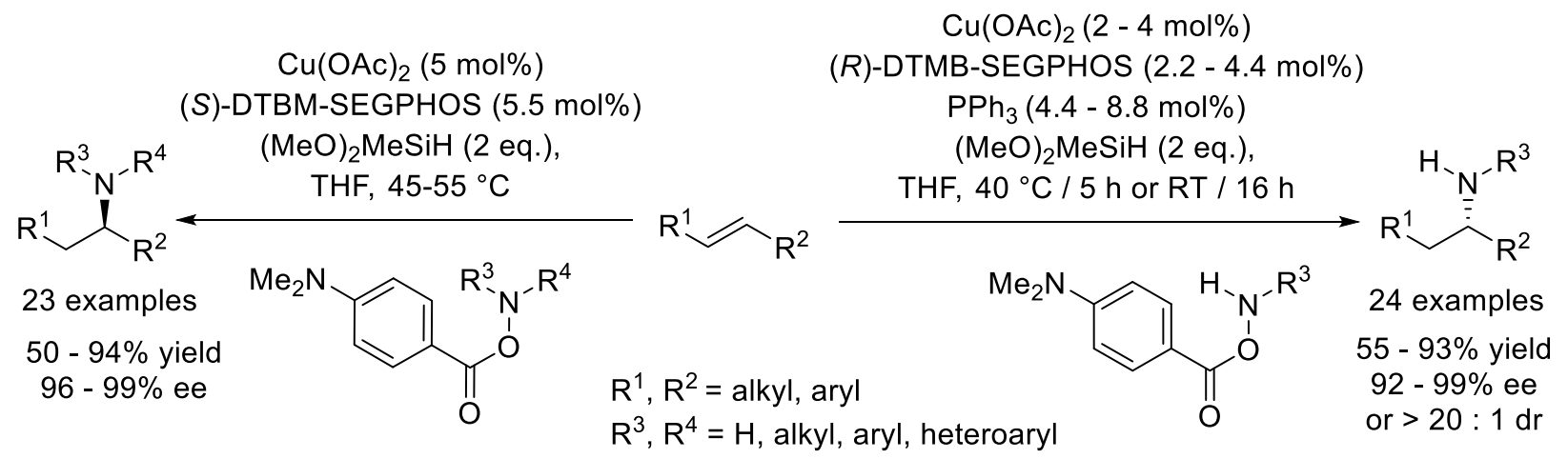

Scheme 22. Optimised copper-hydride catalysed formal hydroamination of internal olefins

For such less reactive substrates, the competitive and non-productive consumption of dialkylhydroxylamine esters was suppressed through the use of more electron-rich benzoates. A fine tuning of the electronic nature of the latter afforded the optimal electrophilic amination agents. Thus, a broad range of amine transfer reagents, i.e. hydroxylamine esters bearing a 4$N, N$-dimethylbenzoate unit, was examined for the formal hydroamination of trans-2-butene. The corresponding tertiary amines were obtained in high yields and enantioselectivities (Scheme 22), demonstrating therefore the great tolerance for a large range of sensitive groups. This procedure was then applied to the formal hydroamination of a significant scope of commercially available internal olefins with equal success [32]. In addition, the catalytic system allowed also the preparation of $\beta$-deuteroamines and the late-stage modification of advanced highly functionalised synthetic intermediates.

A full description of the optimisation of this promising copper(I)-hydride $(\mathrm{Cu}-\mathrm{H})$ catalysed formal hydroamination was published by Buchwald et al. (Scheme 22) [33]. The design and application of a series of modified amine transfer reagents was studied in order to circumvent the competitive consumption of the latter by the $\mathrm{Cu}-\mathrm{H}$ species. The extent of this unwanted side 
reaction was shown to depend on the reactivity of the olefinic substrate and, interestingly, the high enantioselectivities were preserved in all cases. As mentioned above, the development of electron-rich benzoate amine transfer reagents provided electrophilic amine sources with less susceptibility to direct reaction with $\mathrm{Cu}-\mathrm{H}$. The use of a 4-(dimethylamine)benzoate group allowed to convert a large scope of styrene substrates ( $\beta$-substituted, $o$ - or $p$-substituted, donating or withdrawing, heterocyclic substituents, bromide) into NHBn containing secondary amines this time $\left(100 \%\right.$ conversion $5 \mathrm{~h}$ at $40{ }^{\circ} \mathrm{C}$ or $16 \mathrm{~h}$ at room temperature with $92 \%$ enantiomeric excess or higher). The scope of amine transfer reagents was quite large comprising carbohydrates, steroids and amino acid esters. In such cases, the diastereoselective transformation was under chiral catalyst control and no epimerization of chiral $\alpha$-amino acids was observed. The usefulness of this methodology has been exemplified in the synthesis of drug molecules (Sensipar) and commercial pharmaceutical derivatisations (chlorprozamine, loratidine, tufnil). Hence, because of its high chemoselectivity for olefins and its tolerance to sensitive functional groups, the catalytic system developed by Buchwald et al. was thus versatile. Triphenylphosphine could be used as a secondary ligand in order to reduce the stoechiometry of the chiral ligand as respect to the copper pre-catalyst loading (vide infra).

As an extension of this approach, the preparation of amines bearing remote stereocentres was developed by Buchwald et al. by applying the chiral $\mathrm{Cu}-\mathrm{H}$ based catalytic system to the transformation of allylic-alcohols, -ethers and -esters in the presence of electrophilic amine transfer agents [34]. In this case, the reaction mechanism did not rely on a formal hydroamination across the $\mathrm{C}=\mathrm{C}$ bond, but on a reductive relay hydroamination which introduced the amino group remote from the stereocentre created during the enantioselective hydrocupration of the $\mathrm{C}=\mathrm{C}$ bond. Indeed, after the hydrocupration step, a $\beta$-alkoxide elimination provided a new $\mathrm{C}=\mathrm{C}$ bond which could undergo an anti-Markovnikow hydroamination affording the terminal amine with a remote carbon stereocentre.

Latter, Buchwald et al. reported also on the understanding of the factors governing this copper hydride catalysed formal hydroamination reaction [35]. They performed a thorough investigation of the $\mathrm{Cu}-\mathrm{H}$ catalysed formal hydroamination of styrene with extensive use of kinetics, spectroscopies as well as nonlinear effect and deuterium-labelling experiments. A modified catalytic cycle was proposed based on reaction profile analysis, rate order assessment, and Hammett studies (Scheme 23). 


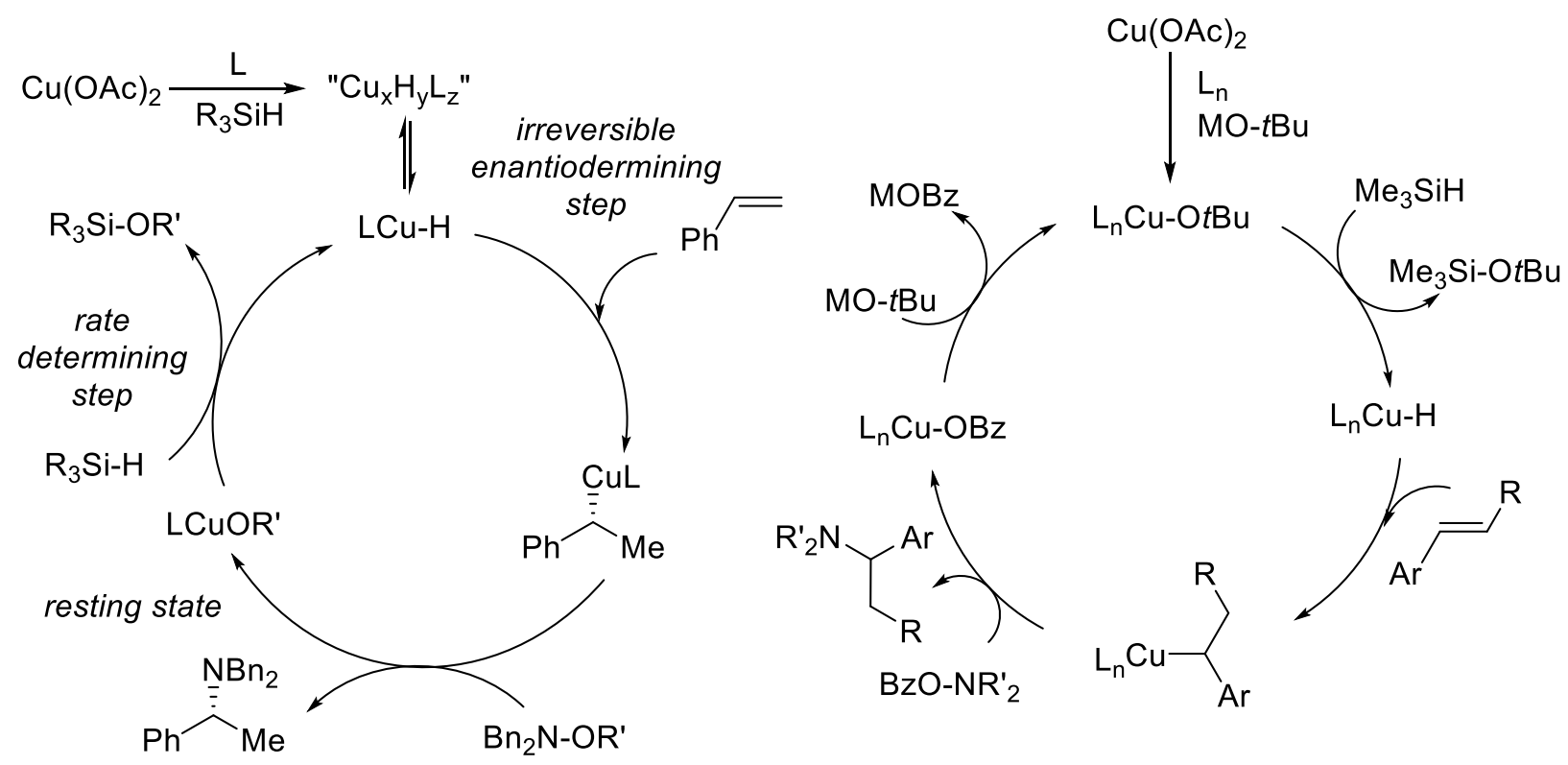

Mechanism proposed by Buchwald Mechanism proposed by Miura

Scheme 23. Proposed reaction mechanisms for the copper-hydride catalysed formal hydroamination of alkenes

Key propositions were: 1) the turnover limiting step was the regeneration of the $\mathrm{Cu}-\mathrm{H}$ species in the presence of the silane, 2) the phosphine coordinated copper(I) benzoate was the catalyst resting state, 3) the catalyst was most probably a monomeric species, 4) the enantiodetermining step was the hydrocupration of the alkene. The rate of the transformation could be improved with judicious choice of silane, amine electrophile, and secondary phosphine ligand additive. A final optimisation of the reaction allowed formal hydroamination of alkenes to be performed through a simple experimental procedure, under air with short reaction times while keeping high yields and enantioselectivities (Scheme 24).

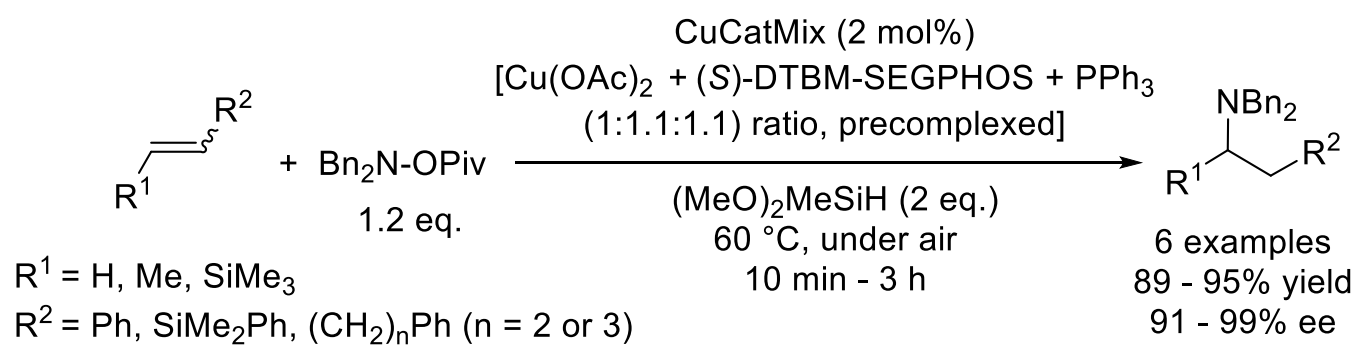

Scheme 24. Optimised copper-hydride catalysed formal hydroamination of alkenes 
In parallel, Hirano and Miura proposed a different mechanistic working hypothesis (Scheme 23) [27]. By comparison to the catalytic system developed by Buchwald which involved the direct reaction of the silane with the $\mathrm{Cu}-\mathrm{H}$ species, they proposed a catalytic system involving $\mathrm{LiO} t \mathrm{Bu}$ as a base. The latter operated first a transmetallation with $\mathrm{Cu}-\mathrm{OBz}$ to lead to $\mathrm{Cu}-\mathrm{O} t \mathrm{Bu}$ which reacted with the silane $\mathrm{Si}-\mathrm{H}$ and generate the $\mathrm{Cu}-\mathrm{H}$ species. On the basis of the catalytic systems developed respectively by Miura et al. and Buchwald et al., Tobisch studied through a detailed computational approach the mechanism of $\mathrm{Cu}-\mathrm{H}$ catalysed formal hydroamination of styrene [36]. The calculation results suggested a catalytic cycle which was in very good agreement with the Buchwald proposal [35] and which differed from Miura's proposal. Indeed, the regeneration of the $\mathrm{Cu}-\mathrm{H}$ catalytic species did not require a large excess of base. However, as already mentioned above, the reactions could not be considered as strict hydroaminations due to their electrophilic pathway and their alkoxysilane by-products (Scheme 23, right hand side).

Afterwards, the $\mathrm{Cu}-\mathrm{H}$ formal hydroamination methodology was extended by Hirano and Miura to oxa- and azabicyclic alkenes providing important enantioenriched amines useful for biological applications (Scheme 25) [37].
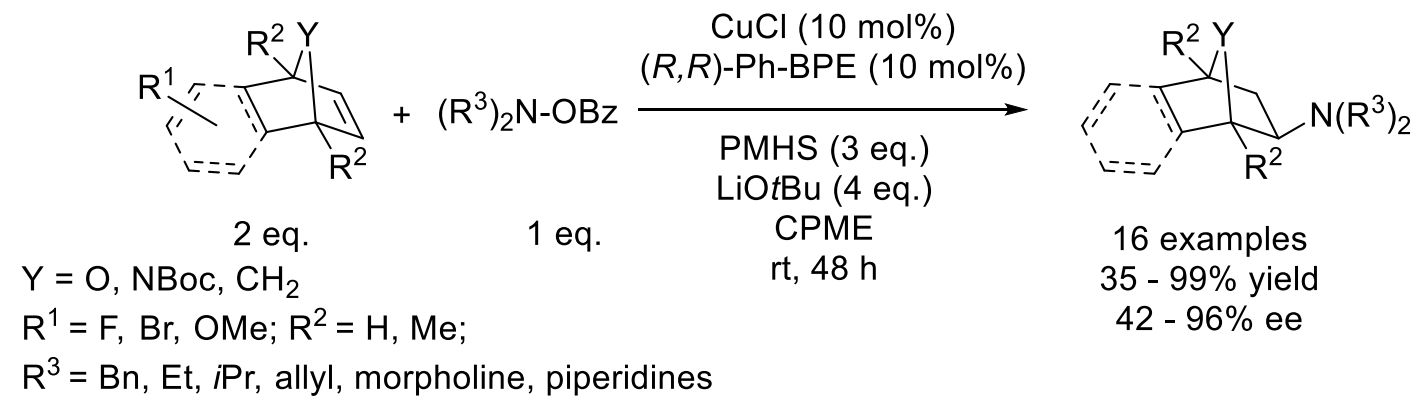

Scheme 25. Copper catalysed formal hydroamination of oxa- and azabicyclic alkenes

In addition, Miura et al. applied their catalytic system to the asymmetric synthesis of $\alpha$ aminoboronic acid derivatives (Scheme 26) [38].

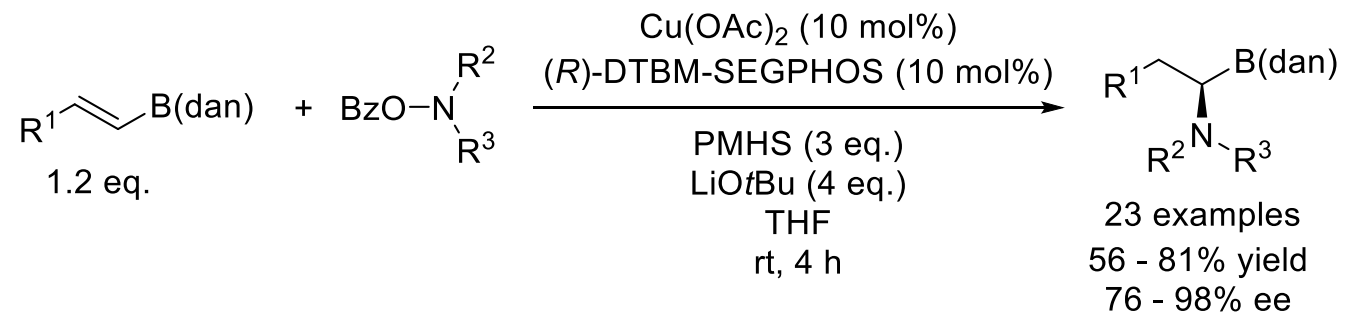

Scheme 26. Copper catalysed formal hydroamination of alkenyl 1,8-diaminonaphthyl) boronate 
By using the alkenyl dan (dan = 1,8-diaminonaphthyl) boronates, the difficult construction of chiral centres that contained unactivated alkyl side chains at the $\alpha$ position was possible in a regio- and enantioselective manner. The regioselectivity was proposed to rely on the hyperconjugation existing between the $\mathrm{Cu}-\mathrm{C} \alpha$ bond and the empty $\mathrm{p}$ orbital on boron within the catalytic intermediate as the result of the first step of the catalytic cycle i.e. the hydrocupration of the $\mathrm{C}=\mathrm{C}$ substrate bond.

Copper-hydride catalysed formal hydroamination was recently applied by Hartwig et al. to unactivated internal alkenes bearing a directing group at the homoallylic position (Scheme 27) [39].

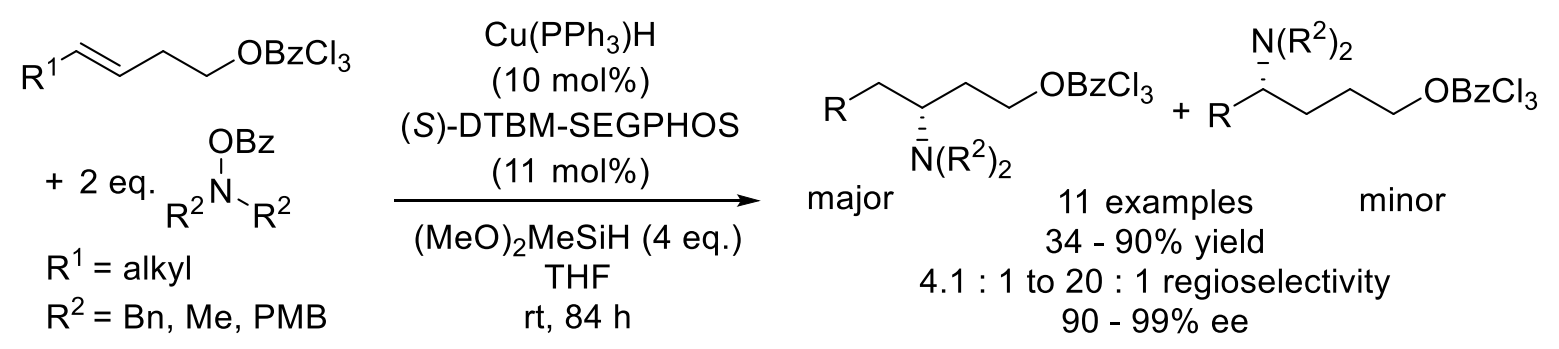

Scheme 27. Copper catalysed formal hydroamination of internal alkenes bearing a directing group

A study of various directing groups allowed to identify the 2,4,6-trichlorobenzoyl $\left(\mathrm{BzCl}_{3}\right)$ as the most able to suppress the competitive reduction of the ester directing group itself. Nevertheless, depending on the substrate, other directing groups like ethers, esters and sulphonamides could be beneficial. The reaction proceeded with high yields, good regioselectivities and excellent enantioselectivities without any excess of alkene reagent. If the directing group was shown to control the regioselectivity through electronic effects, the chiral catalyst could control the reaction diastereoselectivity when a chiral homoallylic ester was reacted. However, a poor regioselectivity was obtained either when the alkene was positioned one carbon further away, or when it was of cis-configuration.

Recently, chiral intramolecular hydroaminations were studied using other copper based catalytic systems. First, Chemler et al., who reported in 2010 an asymmetric alkene diamination catalysed by a $\mathrm{Cu}-(R, R)-\mathrm{Ph}-$ box based complex [40], extended their combination of intra and intermolecular hydroaminations to the highly enantioselective synthesis of 2-aminomethyl 
indolines and pyrrolidines (Scheme 28) [41].<smiles>[X]c1ccc(NS(=O)(=O)c2cc(CC=C)c(OC)c(C(C)(C)C)c2)c(CC(C)(C)C)c1</smiles>

$\mathrm{Cu}(\mathrm{OTf})_{2}(20 \mathrm{~mol} \%)$ ligand (25 mol\%) $\mathrm{R}^{2} \mathrm{NH}_{2}$ (4 - 5 eq.)

or

2,6-di-tBu-4-methylpyridine (1 eq.)

$\mathrm{MnO}_{2}(300 \mathrm{~mol} \%)$

$\mathrm{KMnO}_{4}(8-15 \mathrm{~mol} \%)$<smiles>[R][R]([R])(CN)CC=C</smiles>

$\mathrm{X}=\mathrm{H}, \mathrm{OMe}, \mathrm{Br}, \mathrm{Cl}, \mathrm{F}, \mathrm{Me}$
$\mathrm{R}^{1}=\mathrm{SES}, \mathrm{Ts}, \mathrm{Bs}$
$\mathrm{R}^{2}=\mathrm{H}, \mathrm{Me}$<smiles>[R]NCC1Cc2cc([X])ccc2N1</smiles>
$\mathrm{SO}_{2}$<smiles>COc1c(CC(C)C)cc(S(=O)(=O)O)cc1CC(C)C</smiles>

tBu 8 examples $59-63 \%$ yield $81-91 \%$ ee

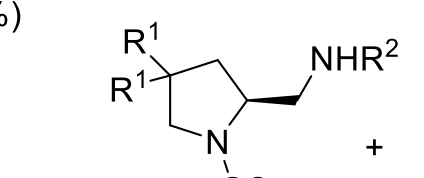

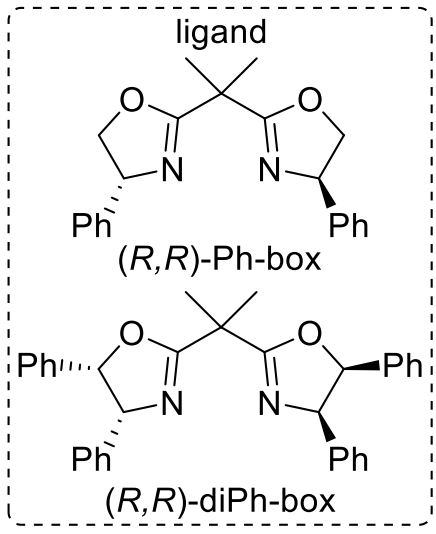

$\mathrm{R}^{1}$<smiles>[R]C1([R])CNC(CCCC(C)(C)C)C1</smiles>

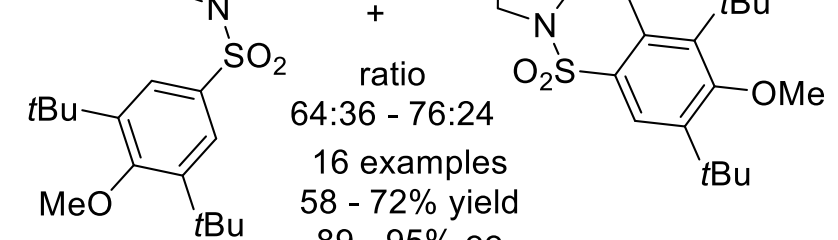
$58-72 \%$ yield $89-95 \%$ ee

Scheme 28. Intra and intermolecular asymmetric hydroamination using a chiral copper complex.

The catalytic system was optimised through change of catalyst, base and oxidant at different loadings. Though rather high loadings of copper and ligand were required, the method proved to be efficient for the coupling of a number of external sulphonamide nucleophiles and aminoalkenes affording the targeted 2-aminomethyl indolines and pyrrolidines.

A second interesting intramolecular hydroamination mediated by sub-stoichiometric amounts of a chiral copper complex was reported by Zeng et al. For the first time, intramolecular double hydroamination of $\mathrm{N}$-alkenylureas was performed and allowed the assembly of chiral vicinal diamino bicyclic heterocycles (Scheme 29) [42].
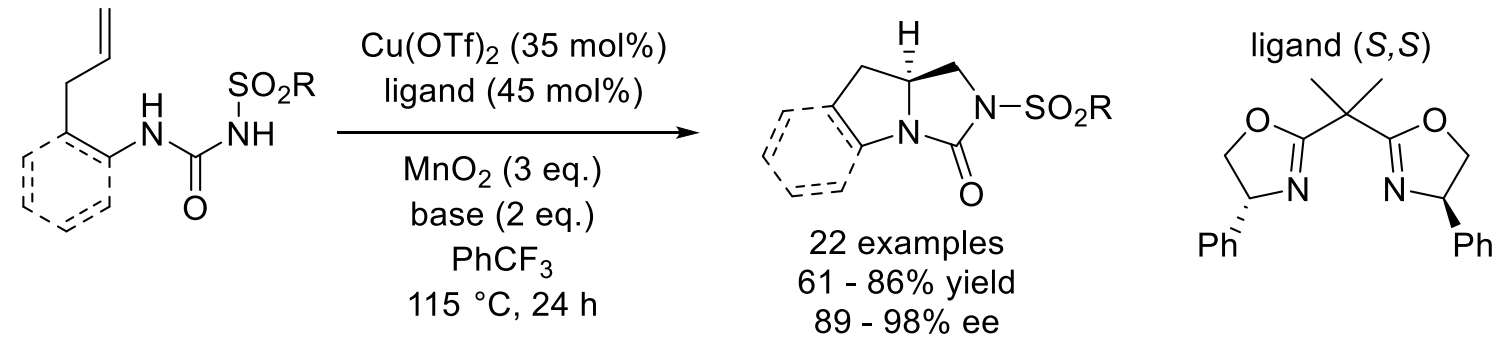

Scheme 29. Intramolecular double hydroamination of $N$-alkenylureas using sub-stoichiometric amounts of a chiral copper complex 


\subsection{Gold catalysts.}

In 2013, Shi et al. reported on the preparation of several monodentate axially-chiral $\mathrm{N}$ heterocyclic carbene and phosphine ligands for the mononuclear gold(I) catalysed intramolecular hydroamination of $N$-alkenyl tosylates (Scheme 30) [43].
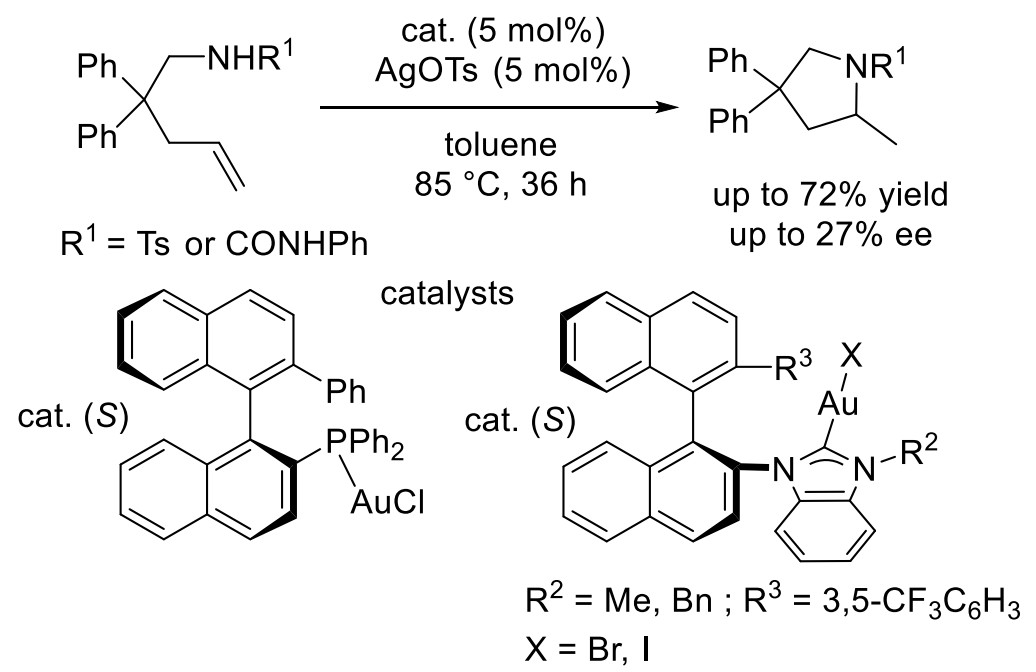

Scheme 30. Asymmetric intramolecular hydroamination of alkenes using gold(I) catalysts based on monophosphine and aminocarbene ligands by Shi et al.

Good yields and moderate enantioselectivities were obtained at quite high temperatures and reaction times. Interestingly, the authors studied their gold(I) pre-catalysts by X-ray diffraction analyses and identified a weak gold- $\pi$ interaction between the gold atom and one of the ligand aromatic ring. Such interactions to aromatic rings of the ligand have been observed before [44] and might also occur with the gold cationic catalyst in the course of the reaction.

Following their ongoing interest in hydroamination reactions [45], Agbossou, Michon et al. studied the intramolecular gold(I) catalysed asymmetric hydroamination of alkenes by screening a wide range of monophosphine and aminocarbene ligands [46a]. Specially designed phosphoramidite ligands proved to lead to active mononuclear gold(I) catalysts when combined with silver salts in toluene. Indeed, chiral amines were obtained in high yields and average enantioselectivities using mild reaction conditions (Scheme 31, A).

Afterwards, Agbossou, Michon et al. studied various binuclear gold(I) catalysts based on diphosphine ligands. When combined with selected silver salts, a gold(I) species built on a 
specific SEGPHOS diphosphine ligand proved to perform efficiently the intramolecular hydroamination of alkenes at mild temperatures with high yields and enantioselectivities (Scheme 31, B) [46b]. Most interestingly, the addition of up to ten equivalents of water to the reaction medium proved to be critical in order to significantly enhance yields and enantioselectivities. Though these reactions are still under investigation, the propensity of perchlorate and tetrafluroborate salts to increase the surface tension of water and, at the meantime, to act as salting-in agents might explain a better solvation of catalyst and organic molecules [47]. The reaction started first by the nucleophilic attack which was previously found to be reversible (Scheme 31) [48]. Second, the tautomerization of the resulting intermediate was likely to be assisted by the anion [49a]. Finally, the stereochemical outcome of the hydroamination reaction appeared to be defined during the final irreversible protodeauration step [48] by differentiation of the two diastereomeric intermediates whose structures depended on the polarity of the solvent used. Because any gold assisted Brønsted acid catalysis [50] was in this case unlikely, the methanol could act as a proton-transfer agent [49b-c], similar in feature to that of anions [49a]. Moreover, as opposed to urea amino-alkenes [51], a bimetallic activation of carbamate substrates could not be evidenced yet. Because a single gold atom shall be sufficient to activate the alkene function towards the nucleophilic attack, the participation of the second gold atom to the hydroamination reaction can be here questionable. Such an issue was already adressed for the bis(gold) catalysed asymmetric intermolecular hydroamination of allenes [52]. According these authors, the decreasing enantioselectivities of the reaction product combined with increasing conversions may be the result of the increasing concentration of a less selective ligated catalyst species. For Agbossou, Michon et al, such a change of enantioselectivity was not observed for the gold catalysed intramolecular hydroamination of alkenes and the role of the second gold atom remains to be understood. 

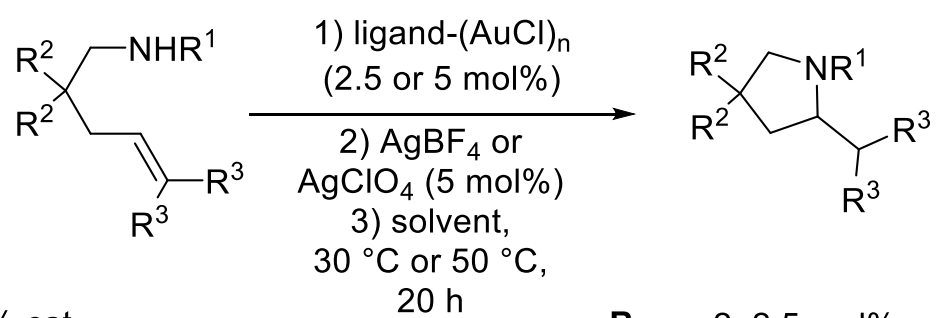

$30^{\circ} \mathrm{C}$ or $50^{\circ} \mathrm{C}$

A: $\mathrm{n}=1,5 \mathrm{~mol} \%$ cat.

B: $n=2,2.5 \mathrm{~mol} \%$ cat.

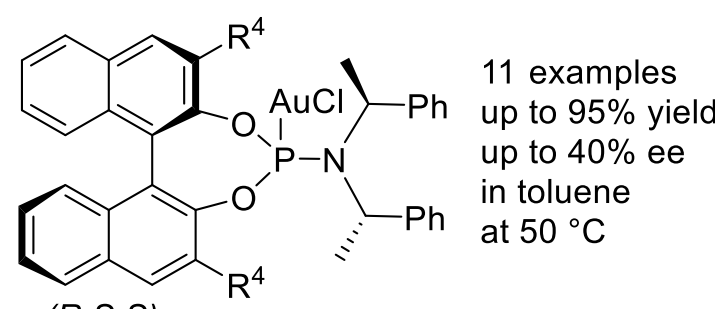

$(R, S, S)$

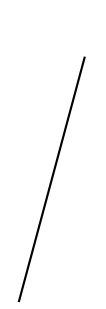

$\mathrm{R}^{1}=\mathrm{CBz}$, Boc, Fmoc, $\mathrm{CO}_{2} \mathrm{Me}, \mathrm{CONHPh} . .$.

$\mathrm{R}^{2}=\mathrm{Ph},-\left(\mathrm{CH}_{2}\right)_{5^{-}}, \mathrm{Me}, \mathrm{H}$

$\mathrm{R}^{3}=\mathrm{Bn}, \mathrm{Me}, \mathrm{H}$

$\mathrm{R}^{4}=\mathrm{H}, \mathrm{Ph}, 4-(\mathrm{Ph})-\mathrm{C}_{6} \mathrm{H}_{4^{-}}, \mathrm{CH}(\mathrm{Ph})_{2} \ldots$

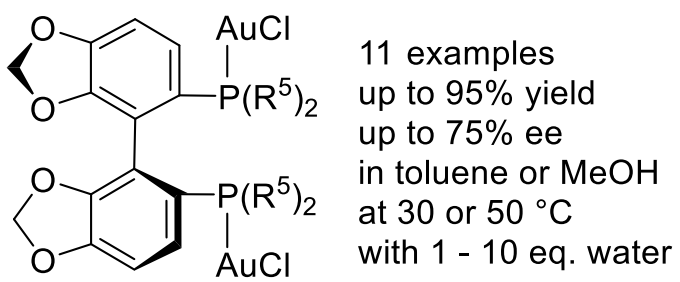

(S) with 1 - 10 eq. water

tBu

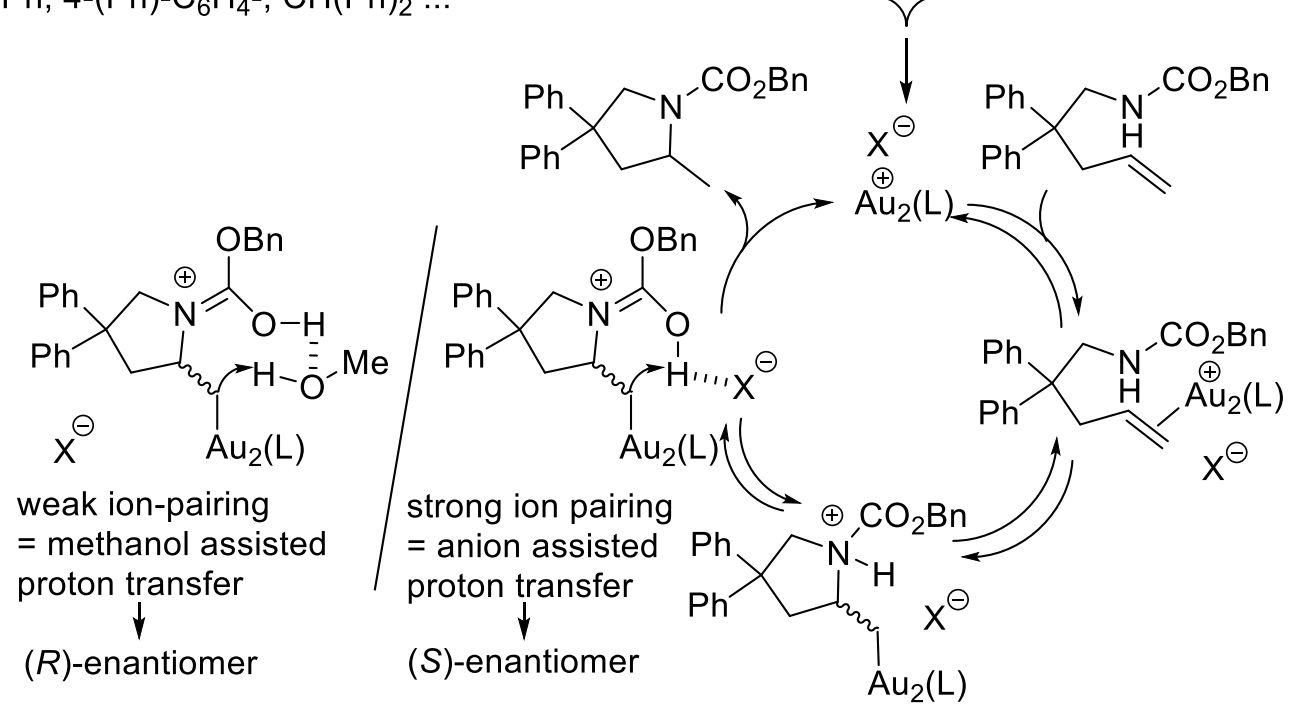

Scheme 31. Asymmetric intramolecular hydroamination of alkenes using gold(I) catalysts based on phosphoramidite and SEGPHOS-type ligands by Agbossou, Michon et al.

However, recent works have reported on the silver salt effects on gold catalysed reactions. Positive or negative impacts on conversions and selectivities were observed whereas the interference of silver and chloride has been highlighted by several bonding modes with gold complexes [53]. While studying intramolecular asymmetric hydroamination of alkenes, Agbossou, Michon et al. noticed a positive silver effect for binuclear gold catalysts but none for mononuclear ones [46]. Hence, they assumed the binuclear catalyst's second gold atom may 
interfere positively with silver chloride within the catalytic process depending on the solvent used [46c].

Shortly after, Widenhoefer et al. reported the intramolecular hydroamination of $\mathrm{N}$-4pentenylcarbamates and ureas catalysed by the combination of a mono- or binuclear gold complex and AgOTf (5 mol\%) in methanol (Scheme 32) [54].

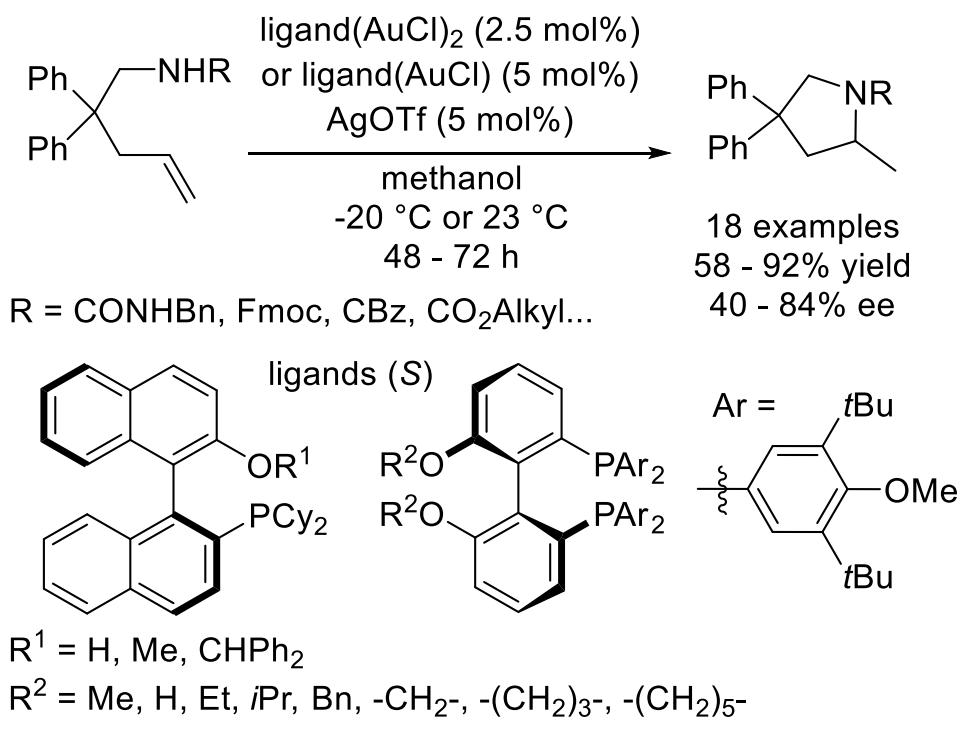

Scheme 32. Asymmetric intramolecular hydroamination of alkenes using gold(I) catalysts based on MOP and BIPHEP ligands by Widenhoefer et al.

While using a binuclear gold catalyst based on (S)-DTBM-MeO-BIPHEP, average to high yields and enantioselectivities were obtained at room temperature, $0{ }^{\circ} \mathrm{C}$ or $-20{ }^{\circ} \mathrm{C}$ along 2 or 3 days of reaction. Besides the axially chiral bidentate BIPHEP ligands, monodentate phosphines derivatised from MOP ligand skeleton led to active mononuclear gold catalysts which allowed quantitative reactions but low enantioselectivities. If BIPHEP based binuclear gold catalysts were highly enantioselective for the intermolecular hydroamination of 1-alkenes with imidazolidin-2-ones using aprotic and apolar aromatic solvents, they only allowed enantioselective intramolecular hydroamination of $\mathrm{N}$-4-pentenylcarbamates and ureas when methanol was used as a solvent. According the authors, such marked difference in optimal 
reaction medium reflected different stereochemical determining steps in the two organic transformations. Indeed, whereas the protodeauration step in a non-polar solvent would occur through a non-selective two-step process involving intermolecular deprotonation/protodeauration, the latter may occur in methanol through a stereoselective onestep process involving concerted intramolecular proton transfer via a hydrogen-bonded methanol bridge [49b].

\section{Conclusion}

The asymmetric hydroamination of alkenes still constitutes an elegant and straightforward way to prepare chiral amines. There's no doubt the major contributions highlighted in this review will motivate future research to implement this synthetic methodology. Indeed, the application of new original chiral organometallic catalysts based on alkaline earth, group 4 metals, late transition metals as well as lanthanides has allowed reaching promising catalytic activities and enantioselectivities. If the control of the stereoselectivity remains a challenge in several cases, further developments of such interesting catalysts are foreseen.

Since 2013, the most significant progress has been undoubtedly the combined use of chiral copper-hydride catalysts and electrophilic amine transfer reagents. By operating through hydrocupration of the alkene substrates and further electrophilic amination, the developed catalytic systems have allowed the efficient formal asymmetric hydroamination of a wide range of substrates with various functional groups. Because the reactions could be implemented under air with high activities and stereocontrol levels, this methodology shall find in the future extensive applications for the synthesis of key amine compounds.

\section{Acknowledgements}

This work was first financed by the French National Research Agency with project ANR-09BLAN-0032-02 (with a PhD fellowship to F.M). The University of Lille 1 is gratefully acknowledged for a PhD fellowship to M.A.A. The CNRS, the Chevreul Institute (FR 2638), the Ministère de l'Enseignement Supérieur et de la Recherche, the Région Nord - Pas de Calais and the FEDER are acknowledged for supporting and funding partially this work.

\section{References}

[1] (a) K. S. Hayes, Appl. Catal. A: Gen. 221 (2001) 187-195; 
(b) S. A. Lawrence, in Amines: Synthesis, Properties and Applications, Cambridge University Press, 2004;

(c) T. C. Nugent, M. El-Shazly, Adv. Synth. Catal. 352 (2010) 753-819;

(d) A. F. Abdel-Magid, S. J. Mehrman, Org. Process Res. Dev. 10 (2006) 971-1031;

(e) Q. Yang, Q. Wang, Z. Yu, Chem. Soc. Rev. 44 (2015) 2305-2329;

(f) M. Breuer, K. Ditrich, T. Habicher, B. Hauer, M. Keßeler, R. Stürmer, T. Zelinski, Angew. Chem. Int. Ed. 43 (2004) 788-824;

(g) M. D. Truppo, J. D. Rozzell, N. J. Turner, Org. Process Res. Dev. 14 (2010) 234-237.

[2] (a) T. E. Müller, M. Beller, Chem. Rev. 98 (1998) 675-704;

(b) J. J. Brunet, D. Neibecker in Catalytic Heterofunctionalization from Hydroamination to Hydrozirconation (Eds.: A. Togni, H. Grutzmacher), Wiley-VCH: Weinheim, Germany, 2001, pp. 10 ;

(c) T. E. Müller, K. C. Hultzsch, M. Yus, F. Foubelo, M. Tada, Chem. Rev. 108 (2008) 3795-3892;

(d) S. Doye in Science of Synthesis, Vol. 40a (Eds.: D. Enders, E. Schaumann), Thieme, Stuttgart, 2009, pp. 241;

(e) J. Hannedouche, E. Schulz, Chem. Eur. J. 19 (2013) 4972-4985;

(f) A. D. Sadow, in Comprehensive Inorganic Chemistry II, J. Reedijk and K. Poeppelmeier (Eds), 2013, vol 6, 487-520;

(g) N. Nishina, Y. Yamamoto, Top. Organomet. Chem. 43 (2013) 115-143;

(h) A. L. Reznichenko, A. J. Nawara-Hultzsch, K. C. Hultzsch, Top. Curr. Chem. 343 (2014) 191260 ;

(i) V. Rodriguez-Ruiz, R. Carlino, S. Bezzenine-Lafollée, R. Gil, D. Prim, E. Schulz, J. Hannedouche, Dalton Trans. 44 (2015) 12029-12059;

(j) E. Bernoud, C. Lepori, M. Mellah, E. Schulz, J. Hannedouche, Catal. Sci. Technol. 5 (2015) 2017-2037;

(k) L. Huang, M. Arndt, K. Gooßen, H. Heydt, L. J. Gooßen, Chem. Rev. 115 (2015) 2596-2697;

(1) L. C. Wilkins, R. L. Melen, Coord. Chem. Rev. 324 (2016) 123-139.

[3] (a) Y. Zhu, R. G. Cornwall, H. Du, B. Zhao, Y. Shi, Acc. Chem. Res. 47 (2014) 3665-3678;

(b) K. Muñiz, C. Martínez, J. Org. Chem. 78 (2013) 2168-2174;

(c) S. De Jong, D. G. Nosal, D. J. Wardrop, Tetrahedron 68 (2012) 4067-4105;

(d) F. Cardona, A. Goti, Nature Chemistry 1 (2009) 269-275.

[4] (a) Mg: X. Zhang, T. J. Emge, K. C. Hultzsch, Angew. Chem. Int. Ed. 51 (2012) 394 -398;

(b) Mg: X. Zhang, S. Tobisch, K. C. Hultzsch, Chem. Eur. J. 21 (2015) 7841-7857;

(c) Ca: J. Penafiel, L. Maron, S. Harder, Angew. Chem. Int. Ed. 54 (2015) 201-206; 
(d) Ca: S. Harder, Chem. Rev. 110 (2010) 3852-3876;

(e) S. Tobisch, Chem. Eur. J. 20 (2014) 8988-9001.

[5] B. Schmid, S. Frieß, A. Herrera, A. Linden, F. W. Heinemann, H. Locke, S. Harder, R. Dorta, Dalton Trans. 45 (2016) 12028-12040.

[6] A. L. Reznichenko, K. C. Hultzsch, Organometallics 32 (2013) 1394-1408.

[7] (a) J. Y. Kim, T. Livinghouse, Org. Lett. 7 (2005) 1737-1739;

(b) Y. Zhang, W. Yao, H. Li, Y. Mu, Organometallics 31 (2012) 4670-4679.

[8] Y. Chapurina, R. Guillot, D. Lyubov, A. Trifonov, J. Hannedouche, E. Schulz, Dalton Trans. 42 (2013) 507-520.

[9] (a) I. Aillaud, J. Collin, C. Duhayon, R. Guillot, D. Lyubov, E. Schulz, A. Trifonov, Chem. Eur. J. 14 (2008) 2189-2200;

(b) A. Mukherjee, S. Nembenna, T. K. Sen, S. Pillai Sarish, P. K. Ghorai, H. Ott, D. Stalke, S. K. Mandal, H. W. Roesky, Angew. Chem. Int. Ed. 50 (2011) 3968-3972.

[10] S. Germain, E. Schulz, J. Hannedouche, ChemCatChem 6 (2014) 2065-2073.

[11] S. D. Bennett, B. A. Core, M. P. Blake, S. J. A. Pope, P. Mountford, B. D. Ward, Dalton Trans. 43 (2014) 5871-5885.

[12] About Thorpe-Ingold effects in hydroamination reactions:

(a) A. Zulys, M. Dochnahl, D. Hollmann, K. Löhnwitz, J.-S. Herrmann, P. W. Roesky, S. Blechert, Angew. Chem. Int. Ed. 44 (2005) 7794-7798;

(b) J. Koller, R. G. Bergman, Chem. Commun. 46 (2010) 4577-4579.

[13] (a) K. Huynh, T. Livinghouse, H. M. Lovick, Synlett 25 (2014)1721-1724;

(b) K. Huynh, B. K. Anderson, T. Livinghouse, Tetrahedron Lett. 56 (2015) 3658-3661.

[14] Z. Chai, D. Hua, K. Li, J. Chu, G. Yang, Chem. Commun. 50 (2014) 177-179.

[15] (a) M. J. Calhorda, C. C. Romao, L. F. Veiros, Chem. Eur. J. 8 (2002) 868-875;

(b) L. F. Veiros, M. J. Calhorda, Dalton Trans. 40 (2011) 11138-11146;

(c) L. Orian, L. P. Wolters, F. M. Bickelhaupt, Chem. Eur. J. 19 (2013) 13337-13347;

(d) R. W. Baker, P. Turner, I. J. Luck, Organometallics 34 (2015) 1751-1758.

[16] (a) (Ti): J. Okuda, P. Koenig, I. L. Rushkin, H.-C. Kang, W. Massa, J. Organomet. Chem. 501 (1995) 37-9;

(b) (Zr): C. A. Bradley, I. Keresztes, E. Lobkovsky, P. J. Chirik, Organometallics 25 (2006) 20802089.

[17] H.-L. Teng, Y. Luo, B. Wang, L. Zhang, M. Nishiura, Z. Hou, Angew. Chem. Int. Ed. 55 (2016) 15406-15410. 
[18] a) T. S. Brunner, P. Benndorf, M. T. Gamer, N. Knöfel, K. Gugau, P. W. Roesky, Organometallics 35 (2016) 3474-3487; b) S. T. Brunner, L. Hartenstein, P. W. Roesky, J. Organomet. Chem. 730 (2013) 32-36.

[19] L. Hussein, N. Purkait, M. Biyikal, E. Tausch, P. W. Roesky, S. Blechert, Chem. Commun. 50 (2014) 3862-3864.

[20] K. Manna, W. C. Everett, G. Schoendorff, A. Ellern, T. L. Windus, A. D. Sadow, J. Am. Chem. Soc. 135 (2013) 7235-7250.

[21] K. Manna, N. Eedugurala, A. D. Sadow, J. Am. Chem. Soc. 137 (2015) 425-435.

[22] N. Yonson, J. C.-H. Yim, L. L. Schafer, Inorganica Chimica Acta 422 (2014) 14-20.

[23] X. Zhou, B. Wei, X.-L. Sun, Y. Tang, Z. Xie, Chem. Commun. 51 (2015) 5751-5753.

[24] G. Sipos, A. Ou, B. W. Skelton, L. Falivene, L. Cavallo, R. Dorta, Chem. Eur. J. 22 (2016) 69396946.

[25] F. Yu, P. Chen, G. Liu, Org. Chem. Front. 2 (2015) 819-822.

[26] Z. Yang, C. Xia, D. Liu, Y. Liu, M. Sugiya, T. Imamoto, W. Zhang, Org. Biomol. Chem. 13 (2015) 2694-2702.

[27] Y. Miki, K. Hirano, T. Satoh, M. Miura, Angew. Chem. Int. Ed. 52 (2013) 10830-10834.

[28] M. T. Pirnot, Y.-M. Wang, S. L. Buchwald, Angew. Chem. Int. Ed. 55 (2016) 48-57.

[29] S. Zhu, N. Niljianskul, S. L. Buchwald, J. Am. Chem. Soc. 135 (2013) 15746-15749.

[30] These important results have been highlighted: K. D. Hesp, Angew. Chem. Int. Ed. 53 (2014) 2035-2036.

[31] S. Zhu, S. L. Buchwald, J. Am. Chem. Soc. 136 (2014) 15913-15916.

[32] Y. Yang, S.-L. Shi, D. Niu, P. Liu, S. L. Buchwald, Science 349 (2015) 62-66.

[33] D. Niu, S. L. Buchwald, J. Am. Chem. Soc. 137 (2015) 9716-9721.

[34] S. Zhu, N. Niljianskul, S. L. Buchwald, Nat. Chem. 8 (2016) 144-149.

[35] J. S. Bandar, M. T. Pirnot, S. L. Buchwald, J. Am. Chem. Soc. 137 (2015) 14812-14818.

[36] S. Tobisch, Chem. Eur. J. 22 (2015) 8290-8300.

[37] Y. Miki, K. Hirano, T. Satoh, M. Miura, Org. Lett. 16 (2015) 1498-1501.

[38] D. Nishikawa, K. Hirano, M. Miura, J. Am. Chem. Soc. 137 (2016) 15620-15623.

[39] Y. Xi, T. W. Butcher, J. Zhang, J. F. Hartwig, Angew. Chem. Int. Ed. 55 (2016) 776-780.

[40] F. C. Sequeira, B. W. Turnpenny, S. R. Chemler, Angew. Chem. Int. Ed. 49 (2010) 6365-6368.

[41] B. W. Turnpenny, S. R. Chemler, Chem. Sci. 5 (2014) 1786-1793.

[42] S. Fu, H. Yang, G. Li, Y. Deng, H. Jiang, W. Zeng, Org. Lett. 17 (2015) 1018-1021.

[43] Y.-W. Sun, Q. Xu, M. Shi, Beilstein J. Org. Chem. 9 (2013) 2224-2232. 
[44] (a) M. Touil, B. Bechem, A. S. K. Hashmi, B. Engels, M. A. Omary, H. Rabaâ, J. Mol. Structure:Theochem. 957 (2010) 21-25;

(b) P. Pérez-Galán, N. Delpont, E. Herrero-Gómez, F. Maseras, A. M. Echavarren, Chem. Eur. J. 16 (2010) 5324-5332;

(c) A. S. K. Hashmi, B. Bechem, A. Loos, M. Hamzic, F. Rominger, H. Rabaâ, Aust. J. Chem. 67 (2014) 481-499.

[45] (a) C. Michon, F. Medina, F. Capet, P. Roussel, F. Agbossou-Niedercorn, Adv. Synth. Catal. 352 (2010) 3293-3305;

(b) F. Medina, C. Michon, F. Agbossou-Niedercorn, Eur. J. Org. Chem. 2012 (2012) 6218-6227;

(c) F. Medina, N. Duhal, C. Michon, F. Agbossou-Niedercorn, Comptes Rendus Chimie 16 (2013) 311-317;

(d) C. Michon, F. Medina, M.-A. Abadie, F. Agbossou-Niedercorn, Organometallics 32 (2013) 5589-5600.

[46] (a) C. Michon, M.-A. Abadie, F. Medina, F. Agbossou-Niedercorn, Catalysis Today 235 (2014) 213 ;

(b) M.-A. Abadie, X. Trivelli, F. Medina, F. Capet, P. Roussel, F. Agbossou-Niedercorn, C. Michon, ChemCatChem, 6 (2014) 2235-2239;

(c) M.-A. Abadie, X. Trivelli, F. Medina, N. Duhal, M. Kouach, B. Linden, E. Genin, F. Capet, P. Roussel, I. Del Rosal, L. Maron, F. Agbossou-Niedercorn, C. Michon, submitted.

[47] About the Hofmeister series: P. Lo Nostro, B. W. Ninham, Chem. Rev. 112 (2012) 2286-2322.

[48] R. L. LaLonde, W. E. Brenzovich, D. Benitez, E. Tkatchouk, K. Kelley, W. A. Goddard III, F. D. Toste, Chem. Sci. 1 (2010) 226-233.

[49] (a) G. Kovács, G. Ujaque, A. Lledós, J. Am. Chem. Soc. 130 (2008) 853-864;

(b) C. M. Krauter, A. S. K. Hashmi, M. Pernpointner, ChemCatChem 2 (2010) 1226-1230;

(c) J. Zhang, W. Shen, L. Li, M. Li, Organometallics 28 (2009) 3129-3139.

[50] O. Kanno, W. Kuriyama, J. Z. Wang, D. F. Toste, Angew. Chem. Int. Ed. 50 (2011) 9919-9922.

[51] M. Kojima, K. Mikami, Synlett 23 (2012) 57-61.

[52] K. L. Butler, M. Tragni, R. A. Widenhoefer, Angew. Chem. Int. Ed. 51 (2012) 5175-5178.

[53] See also references therein;

(a) J. Han, N. Shimizu, Z. Lu, H. Amii, G. B. Hammond, B. Xu, Org. Lett. 16 (2014) 3500-3503;

(b) W. Fang, M. Presset, A. Guérinot, C. Bour, S. Bezzenine-Lafollée, V. Gandon, Chem. Eur. J. 20 (2014) 5439-5446;

(c) A. Homs, I. Escofet, A. M. Echavarren, Org. Lett. 15 (2013) 5782-5785;

(d) Y. Zhu, C. S. Day, L. Zhang, K. J. Hauser, A. C. Jones, Chem. Eur. J. 19 (2013) 12264-12271; 
(e) D. Wang, R. Cai, S. Sharma, J. Jirak, S; K. Thummanapelli, N. G. Akhmedov, H. Zhang, X. Liu, J. L. Petersen, X. Shi, J. Am. Chem. Soc. 134 (2012) 9012-9019;

(f) H. Schmidbaur, A. Hamel, N. W. Mitzel, A. Schier, S. Nogai, Proc. Natl. Acad. Sci. USA 99 (2002) 4916-4921.

[54] S. D. Lee, J. C. Timmerman, R. A. Widenhoefer, Adv. Synth. Catal. 356 (2014) 3187-3192. 